\title{
Detecting the (Quasi-)Two-Body Decays of $\tau$ Leptons in Short-Baseline Neutrino Oscillation Experiments
}

\author{
A. Asratyan ${ }^{a, *}$ M. Balatz ${ }^{a}$, D. Boehnlein ${ }^{b}$, S. Childres $^{b}$, \\ G. Davidenko ${ }^{a}$, A. Dolgolenko ${ }^{a}$, G. Dzyubenko $^{a}$, \\ V. Kaftanov ${ }^{a}$, M. Kubantsev ${ }^{a, \dagger}$, N. W. Reay ${ }^{c}$, J. Musser $^{d}$,

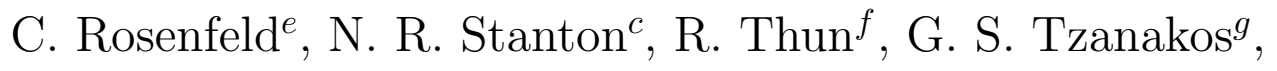 \\ V. Verebryusov ${ }^{a}$, and V. Vishnyakov ${ }^{a}$ \\ (a) Institute of Theoretical and Experimental Physics, 117259 Moscow, Russia \\ (b) Fermi National Accelerator Laboratory, Batavia, IL 60510, USA \\ (c) Kansas State University, Manhattan, KS 66506, USA \\ (d) Indiana University, Bloomington, IN 47405, USA \\ (e) University of South Carolina, Columbia, SC 29208, USA \\ (f) University of Michigan, Ann Arbor, MI 48109, USA \\ (g) University of Athens, 15771 Athens, Greece
}

August 20, 2019 


\begin{abstract}
Novel detector schemes are proposed for the short-baseline neutrino experiments of next generation, aimed at exploring the large- $\Delta m^{2}$ domain of $\nu_{\mu} \rightarrow \nu_{\tau}$ oscillations in the appearance mode. These schemes emphasize good spectrometry for charged particles and for electromagnetic showers and efficient reconstruction of $\pi^{0} \rightarrow \gamma \gamma$ decays. The basic elements are a sequence of relatively thin emulsion targets, immersed in magnetic field and interspersed with electronic trackers, and a fine-grained electromagnetic calorimeter built of lead glass. These elements act as an integral whole in reconstructing the electromagnetic showers. This conceptual scheme shows good performance in identifying the $\tau$ (quasi-)two-body decays by their characteristic kinematics and in selecting the electronic decays of the $\tau$.
\end{abstract}

PACS numbers: 14.60.Pq, 14.60.Fg

Keywords: neutrino oscillations, $\tau$ leptons, nuclear emulsion

${ }^{*}$ Corresponding author. Tel.: (095)-237 0079. E-mail: asratyan@vitep5.itep.ru.

${ }^{\dagger}$ Now at Kansas State University, Manhattan, KS 66506, USA 


\section{Introduction}

Whether or not the leptonic number is strictly conserved is still an open problem of particle physics. The leptonic number may be violated, if the current neutrinos $\nu_{\alpha}(\alpha=e, \mu, \tau)$ are not exactly massless, but rather represent linear combinations of the mass eigenstates $\nu_{i}$ with masses $m_{i}(i=1,2,3): \nu_{\alpha}=U_{\alpha i} \nu_{i}$, where $U_{\alpha i}$ is a unitary mixing matrix that is analogous to the Cabibbo-Kobayashi-Maskawa matrix for the quark sector. Then, as different mass components no longer propagate coherently, one current neutrino may oscillate in flight to another current neutrino [1]: $\nu_{\alpha} \rightarrow \nu_{\beta}, \alpha \neq \beta$. Over a distance $L$ from the point of emission, the transition probability is

$$
P(\alpha \rightarrow \beta)=\delta_{\alpha \beta}-4 \sum_{i>j} U_{\alpha i} U_{\beta i} U_{\alpha j} U_{\beta j} \sin ^{2}\left(\pi L / \lambda_{i j}\right)
$$

where the oscillation length, $\lambda_{i j}$, is proportional to the neutrino energy $E_{\nu}$ and is inversely proportional to the mass difference $\Delta m_{i j}^{2}=m_{i}^{2}-m_{j}^{2}$ :

$$
\lambda_{i j}=4 \pi E_{\nu} \hbar c /\left|\Delta m_{i j}^{2}\right|
$$

Note that in the considered case of just three Dirac neutrinos, we have only two independent mass differences $\Delta m_{i j}^{2}$. Given the values of $E_{\nu}$ and $\Delta m^{2}$, the transition probability reaches its first maximum at a distance $L=1.24 \mathrm{~km} E_{\nu}(\mathrm{GeV}) / \Delta m^{2}\left(\mathrm{eV}^{2}\right)$ from the point of emission.

Searches for neutrino oscillations are actively pursued in experiments with solar, atmospheric, reactor, and accelerator neutrinos. These are characterized by very different $L / E_{\nu}$ ratios and, therefore, are sensitive to very different values of $\Delta m^{2}$. A large deficit of muon neutrinos, as observed in atmospheric showers [2], suggests that they transform to another flavor with $\Delta m^{2}$ on the order of $10^{-2}-10^{-3} \mathrm{eV}^{2}$ and with an effective mixing, 
defined as $\sin ^{2}(2 \theta)=4 U_{\alpha i} U_{\beta i} U_{\alpha j} U_{\beta j}$, between 0.5 and 1.0. On the other hand, an accelerator experiment using neutrinos from $\pi^{+}$and $\mu^{+}$decays at rest [3] has presented evidence for the $\bar{\nu}_{\mu} \rightarrow \bar{\nu}_{e}$ transition with a much larger mass difference of $\Delta m^{2} \sim 1 \mathrm{eV}^{2}$ and with a much smaller effective mixing of $\sin ^{2}(2 \theta) \sim 6 \times 10^{-3}$.

The former pattern of flavor-changing transitions between neutrinos, that is characterized by a small $\Delta m^{2}$ and a large mixing, will be thoroughly investigated in long-baseline accelerator experiments like MINOS at Fermilab [4], in which the muon neutrinos will travel a distance of $731 \mathrm{~km}$ before hitting the detector. The latter pattern of oscillations, that involves $\Delta m^{2} \sim 1 \mathrm{eV}^{2}$ and a small mixing, must be further studied in either the $\nu_{\mu} \rightarrow \nu_{e}$ and $\nu_{\mu} \rightarrow \nu_{\tau}$ channels in short-baseline accelerator experiments that may accumulate sufficient statistics of neutrino interactions.

Despite some appealing attempts to describe all available data as a whole [5, 6], we believe that the experimental situation is still too volatile to allow a coherent and reliable scheme of transitions among the three neutrino flavors. Still, there are sound qualitative reasons to believe that the transition driven by the bigger $\Delta m_{i j}^{2}$ must primarily manifest itself in the $\nu_{\mu} \rightarrow \nu_{\tau}$ channel. Qualitatively, one may expect that the mass hierarchy of neutrinos follows that of corresponding charged leptons, so that $\nu_{\tau}$ should be the most massive. Indeed, the GUT see-saw mechanism [7] predicts that $m_{\nu_{e}} / m_{\nu_{\mu}} / m_{\nu_{\tau}} \sim$ $m_{u}^{2} / m_{c}^{2} / m_{t}^{2}$. On the other hand, in analogy with the known pattern of quark mixings, one may naively expect that mixings between the neighboring neutrino flavors $\left(i . e ., \nu_{e}-\nu_{\mu}\right.$ and $\nu_{\mu}-\nu_{\tau}$ ) should be the strongest, while that for $\nu_{e}-\nu_{\tau}$ may be substantially weaker.

Therefore, if the bigger $\Delta m_{i j}^{2}$ is on an order of $1 \mathrm{eV}^{2}$ as suggested by the data of LSND [3], one should look for the corresponding $\nu_{\mu} \rightarrow \nu_{\tau}$ transition in the short-baseline ( $L \sim 1 \mathrm{~km})$ and medium-baseline $(L \sim 10-20 \mathrm{~km})$ experiments at accelerators. Quite 
importantly, the accelerators like SPS at CERN and Main Injector at Fermilab offer intense and almost pure beams of muon neutrinos with mean energies well above the threshold for $\tau$ production, as required by experiments with $\nu_{\tau}$ appearance.

The flux of $\tau$ neutrinos generated via the flavor-changing transition $\nu_{\mu} \rightarrow \nu_{\tau}$ can only be inferred from the number of $\tau$ leptons produced in the charged-current reaction $\nu_{\tau} N \rightarrow \tau^{-} X$. The created $\tau$ typically travels a distance of one millimeter in space (lifetime $3 \times 10^{-13} \mathrm{~s}$ ) and then decays either leptonically with emission of two neutrinos $\left(\tau^{-} \rightarrow \mu^{-} \bar{\nu} \nu, e^{-} \bar{\nu} \nu\right)$ or hadronically with emission of a single neutrino $\left(\tau^{-} \rightarrow \pi^{-} \nu, \rho^{-} \nu\right.$ $a_{1}^{-} \nu$, etc.). An efficient search for the $\nu_{\mu} \rightarrow \nu_{\tau}$ transition requires that the events of $\tau$ production and decay be selected and analyzed on an event-by-event basis. Therefore, the production and decay vertices must be reliably resolved in the detector (whereby the short track of the $\tau$ is reconstructed in space) and the decay particles must be identified. Of the available detector choices, only nuclear emulsion fully meets the above requirements for a massive target.

The emulsion technique was pioneered by the Fermilab experiment E531 which, in its search for the $\nu_{\mu} \rightarrow \nu_{\tau}$ transition [B] in the appearance mode, found no candidate events of $\tau$ production and decay. For $\Delta m^{2}>50 \mathrm{eV}^{2}$, the mixing parameter $\sin ^{2}(2 \theta)$ was restricted to be less than $5 \times 10^{-3}$. At CERN SPS, the emulsion experiment CHORUS [9] completed operation in 1997, but data processing is still in progress [10]. Provided that no candidate events are finally seen, they will tighten the E531 upper limit on $\sin ^{2}(2 \theta)$ by another order of magnitude. 


\section{Short-Baseline Experiments of Next Generation}

Two short-baseline emulsion experiments of the next generation have been designed to achieve an order-of-magnitude increase in sensitivity over CHORUS [9] and the electronic experiment NOMAD [11]. At Fermilab, E803/COSMOS [12, 13] was optimized for the intense neutrino beam of Main Injector - a $120-\mathrm{GeV}$ proton accelerator to be commissioned in 1999. At CERN, TOSCA [14] will use the $\nu_{\mu}$ beam generated by the $350-\mathrm{GeV}$ proton beam of the SPS accelerator. Like E531 and CHORUS, both are designed as hybrid detectors combining an emulsion target with a downstream electronic spectrometer. The candidates for $\tau$ decays are directly observed in the emulsion target, while the spectrometer is used to select the events to be scanned in emulsion and to measure the momenta of secondary particles for kinematic analysis of $\tau$ candidates.

The baseline schemes of COSMOS and TOSCA are detailed in the respective proposals [13] and [14], and general characteristics of the two experiments are compared in Table 1. Either experiment will collect ten times more CC interactions of muon neutrinos than CHORUS, while rejecting the backgrounds to the $\tau$ signal much more efficiently. The bigger target mass and higher beam energy of TOSCA are seen to be largely compensated by the higher repetition rate of Main Injector that will deliver to target some eight times more protons per year than CERN SPS. As a result, the two experiments may boast comparable sensitivities to the $\nu_{\mu} \rightarrow \nu_{\tau}$ transition in the null limit, that is, assuming that no signals are finally detected.

That the experiment be capable of convincingly interpreting and demonstrating even a relatively small $\tau$ signal, should one show up, is perhaps much more important than just restricting the parameter space for the null hypothesis. For this, an appreciable fraction 
of the signal must be unambiguously reconstructed as $\tau$ decays rather than the decays of anticharm produced by the antineutrino component of the beam. Such distinctive signatures can only be provided by the (quasi-)two-body semileptonic decays of the $\tau$ :

$$
\begin{gathered}
\tau^{-} \rightarrow \pi^{-} \nu(B R=11.3 \%), \\
\tau^{-} \rightarrow \rho^{-} \nu, \rho^{-} \rightarrow \pi^{-} \pi^{0}(B R=25.2 \%), \\
\tau^{-} \rightarrow a_{1}^{-} \nu, a_{1}^{-} \rightarrow \pi^{-} \pi^{+} \pi^{-}(B R=9.4 \%) .
\end{gathered}
$$

In a (quasi-)two-body decay $\tau^{-} \rightarrow h^{-} \nu$, "transverse mass" is defined as $M_{T}=\sqrt{m_{h}^{2}+p_{T}^{2}}+$ $p_{T}$, where $m_{h}$ and $p_{T}$ are the $h^{-}$mass and transverse momentum with respect to $\tau$ direction. The two-body kinematics dictate that the $M_{T}$ distribution should reveal a very distinctive peak just below $M_{T}^{\max }=m_{\tau}$. This "Jacobian cusp", that may provide a characteristic signature of the $\tau$, rapidly degrades with increasing $\Delta p / p$ for the decay products (see [12, 13] and the figures below). Needless to say, analyzing the $\tau^{-} \rightarrow \rho^{-} \nu$ decays also requires good reconstruction of $\pi^{0} \rightarrow \gamma \gamma$ decays in the detector. The $M_{T}$ technique for identifying massive parents by two-body decays in emulsion was proven by observing the relatively rare decays $D_{s}^{+}(1968) \rightarrow \mu^{+} \nu$ against a heavy background from other decays of charm 15].

In its baseline form that emphasizes good spectrometry for charged particles $(\Delta p / p \sim$ 0.03 disregarding the error from multiple scattering in emulsion), COSMOS will be able to observe a distinct Jacobian cusp in the $M_{T}$ distribution for the pionic decay $\tau^{-} \rightarrow$ $\pi^{-} \nu$. The decay $\tau^{-} \rightarrow \rho^{-} \nu$ will be analyzed less efficiently because of difficulties in reconstructing the $\pi^{0} \rightarrow \gamma \gamma$ decays in thick emulsion (three radiation lengths in the baseline design). Thick emulsion will also complicate the analysis of electronic decays

$$
\tau^{-} \rightarrow e^{-} \bar{\nu} \nu(B R=17.8 \%)
$$


and subsequent comparison with the muonic decays

$$
\tau^{-} \rightarrow \mu^{-} \bar{\nu} \nu(B R=17.4 \%)
$$

In TOSCA, the analysis of (quasi-)two-particle decays of the $\tau$ is severely hampered by inferior spectrometry for charged particles $(\Delta p / p \sim 0.1)$ and by the fact that the decays $\pi^{0} \rightarrow \gamma \gamma$ are not reconstructed at all. The one-prong semileptonic decays

$$
\tau^{-} \rightarrow \pi^{-}\left(n \pi^{0}\right) \nu(B R \sim 50 \%)
$$

are treated only inclusively, i.e., without selecting the individual (quasi-)two-particle channels. In this sense, in its baseline form TOSCA is a "counting experiment" that only compares the number of observed kinks with predicted backgrounds from anticharm decays and from pion interactions in emulsion. Thus, despite a somewhat higher sensitivity to the $\nu_{\mu} \rightarrow \nu_{\tau}$ transition in the null limit, TOSCA may have less discovery potential than COSMOS.

\section{Alternative Schemes for COSMOS and TOSCA}

In this paper, we propose alternative conceptual schemes for COSMOS and TOSCA, aimed at enhancing the discovery potentials of both experiments through improving the analysis of (quasi-)two-body decays of the $\tau$. Accordingly, the proposed schemes emphasize good spectrometry for charged particles and electromagnetic showers and efficient reconstruction of $\pi^{0} \rightarrow \gamma \gamma$ decays.

Either scheme involves a sequence of relatively thin emulsion targets, immersed in magnetic field and interspersed with electronic trackers, and a fine-grained electromagnetic calorimeter (EMCal) downstream of the targets. As the total thickness of emulsion 
amounts to several radiation lengths, most photons originating from the targets will produce electromagnetic showers. The adopted strategy is to reassemble the parent photon from unconverted daughter gammas (detected in the EMCal) and from conversion electrons that are momentum-analyzed in the instrumented gaps. By additionally sampling the shower in between the targets that act as converters, we are able to collect a significant fraction of parent energy despite relatively large total thickness of emulsion. Thus, in reconstructing electromagnetic showers the targets, trackers, and the EMCal act as an integral whole. This strategy develops the original philosophy of COSMOS and is very alien to the baseline design of TOSCA in which the six target modules are virtually independent of each other [14].

The strategy of assembling the parent photon from "pieces" dictates that the EMCal should show good performance at energies down to some $100 \mathrm{MeV}$ in a high-multiplicity environment (10-20 showers per event). Of the existing options, these requirements are best met by the technique of Cherenkov lead glass. For either COSMOS and TOSCA, we assume a round-shaped EMCal with 3.5-m diameter, built of lead-glass cells with transverse granularity of 4.25 by $4.25 \mathrm{~cm}$ and with thickness of 15 radiation lengths. The cells are read out by photomultipliers. We have ample experience in constructing and operating very similar devices, and the response of the proposed EMCal is fully understood through extensive simulations and calibrations [16]. It is characterized by an energy resolution of $\delta E(\mathrm{GeV})=0.05 \sqrt{E}+0.02 E$, while the position resolution in either coordinate is close to $2 \mathrm{~mm}$.

In the proposed schemes, as in the baseline design of COSMOS, electronic tracking largely relies on the multisampling (jet) drift chambers that provide excellent pattern recognition, redundancy, and two-track resolution. Following the original design of 
TOSCA, we also include two planes of silicon-microstrip detectors, providing two independent $X Y$ measurements to define a track segment, just downstream of each target (at 10 and $50 \mathrm{~mm}$ from bulk emulsion, respectively). These are primarily aimed at facilitating the extrapolation of tracks into bulk emulsion [17], but also affect the spectrometry of these tracks. In the simulations described in subsequent sections, we assume that the drift chambers and silicon-microstrip planes have spatial resolutions of 150 and $20 \mu \mathrm{m}$, respectively.

In either scheme detailed below, we assume that the targets and trackers are immersed in uniform magnetic field of 0.5 Tesla that is perpendicular to beam direction. The geometry of the magnet is not specified. (Note however that TOSCA will use the existing dipole magnet with useful volume of $3.5 \times 3.5 \times 7.5 \mathrm{~m}^{3}$ and field of up to 0.7 Tesla, that fully meets our requirements. At the same time, our scheme for COSMOS will require a bigger magnet than that foreseen in the original proposal.) Likewise, we do not specify the geometry of the muon identifier that is not relevant to our analysis.

The proposed configuration of COSMOS is shown in Fig. 11. The three emulsion targets with areas of $180 \times 180 \mathrm{~cm}^{2}$ and thicknesses of $3 \mathrm{~cm}(\sim$ one radiation length) are located at $z=0,120$, and $240 \mathrm{~cm}$ along the beam direction. The front face of the EMCal is at $z=540 \mathrm{~cm}$, or far enough from the targets for efficiently reconstructing the $\pi^{0} \rightarrow \gamma \gamma$ decays. Also shown are the pairs of silicon-microstrip planes just downstream of each target and the drift chambers with active area of $240 \times 240 \mathrm{~cm}^{2}$ that instrument the 120-cm-wide gaps between the targets and the 300-cm-wide gap between the downstream target and the EMCal. The total thickness of emulsion is the same as in the baseline scheme $(9 \mathrm{~cm})$, but now we are able to sample electromagnetic showers every radiation length of emulsion. The total amount of emulsion is $1112 \mathrm{~kg}$, or some $30 \%$ more than in 
the baseline design.

For TOSCA, we propose a very similar configuration that is also depicted in Fig. 11. Here, the number of targets is increased to four (at $z=0,150,300$, and $450 \mathrm{~cm}$ ) and their thicknesses are increased to $4 \mathrm{~cm}$, thus bringing the total thickness of emulsion to over five radiation lengths. Compared to COSMOS that will operate at a lower beam energy (see Table 1), the targets are driven farther apart to provide a longer lever arm for momentum analysis. The targets and the drift chambers have the same areas as in the COSMOS configuration $\left(180 \times 180\right.$ and $240 \times 240 \mathrm{~cm}^{2}$, respectively). That the EMCal is now placed farther downstream of the last target (front face at $z=950 \mathrm{~cm}$ ) is dictated by higher mean energy of the $\pi^{0}$ mesons to be reconstructed by TOSCA. Compared to the baseline design of TOSCA, the total amount of emulsion is less by some $17 \%$, while the total area of silicon-microstrip planes is virtually the same.

\section{Simulating $\tau$ Signatures in the Detector}

For either scheme detailed in the previous section, we have performed GEANT-based simulations of detector response to the process of $\tau$ production and decay through various channels. In our calculations, we assume that the energy spectra of incident $\tau$ neutrinos are exactly proportional to those of original $\nu_{\mu}$ beams from the respective accelerators (i.e., from Main Injector for COSMOS and from CERN SPS tuned to 350-GeV proton energy for TOSCA, see Table (1). This corresponds to $\nu_{\mu} \rightarrow \nu_{\tau}$ oscillations driven by a large mass difference $\left(\Delta m^{2}>30\right.$ and $50 \mathrm{eV}^{2}$ for COSMOS and TOSCA, respectively). The radial distribution of either beam at detector position is taken into account. The computed energy spectra of $\nu_{\tau}$-induced $\mathrm{CC}$ interactions for either experiment, that are 
affected by the energy threshold for $\tau$ production, are shown in Fig. 2.

Note that a realistic simulation should include the experimental restrictions dictated by the emulsion technique:

- Because of Coulomb scattering, soft and broad-angle tracks can neither be reconstructed in emulsion nor be linked to the tracker. Therefore, a charged daughter of the $\tau$ can only be detected and momentum-analyzed, if its lab. momentum exceeds some $1 \mathrm{GeV}$ and if its emission angle is within some $400 \mathrm{mrad}$.

- In a one-prong decay (like $\tau^{-} \rightarrow l^{-} \nu \bar{\nu}, \tau^{-} \rightarrow \pi^{-} \nu$, or $\tau^{-} \rightarrow \rho^{-} \nu$ ), the angle between the $\tau$ and charged-daughter directions must exceed some $10 \mathrm{mrad}$ for the "kink" on the $\tau$ track to be detected in emulsion.

- At low transverse momenta, the bulk of detected kinks are due to decays of strange particles and to pion scatterings on the nuclei of emulsion without visible breakup of the nucleus (the so-called "white stars"). These background kinks are largely removed by the cut $p_{T}>250 \mathrm{MeV}$ on transverse momentum of the charged daughter with respect to parent- $\tau$ direction.

A major source of background to the $\tau$ signal is anticharm production in those antineutrino-induced CC events in which the primary charged lepton (either a $\mu^{+}$or a $e^{+}$) has been misidentified in the detector. That the $\tau$, unlike anticharm, is emitted back-to-back with primary hadrons in the transverse plane allows to suppress the anticharm background by additional kinematic cuts (see [12, 13, 14]) that are not discussed in this paper. 


\subsection{Simulation Procedure}

The first stage is to generate the CC collisions of $\tau$ neutrinos with free nucleons, $\nu_{\tau} N \rightarrow$ $\tau^{-} X$, with subsequent leptonic and semileptonic (quasi)-two-particle decays of the emitted $\tau: \tau^{-} \rightarrow l^{-} \nu \bar{\nu}, \pi^{-} \nu, \rho^{-} \nu$, and $a_{1}^{-} \nu$. Both the quasielastic and deep-inelastic processes of $\tau$ production are simulated. For quasielastics, the hadronic tensor is expressed through the known formfactors for the weak $n \rightarrow p$ transition. (Excitation of baryonic resonances like $\Delta^{+}$and $\Delta^{++}$is not simulated explicitly, but is accounted for by simply scaling up by a factor of 2.2 the cross section for $\nu_{\tau} N \rightarrow \tau^{-} N^{\prime}$ on an isospin-averaged nucleon.) For inelastic production of the $\tau$, we rely on the naive parton model.

The process of $\tau$ production and decay is treated as a whole using the narrow-width approximation: for each decay channel, the cross section is computed from an overall Feynman graph with two weak vertices in which $\delta\left(q^{2}-m_{\tau}^{2}\right)$ is substituted for the $\tau$ propagator. In this way, we are able to account for polarization of the $\tau$ that strongly affects the angular distributions of secondary particles in the $\tau$ frame: thus, in the decay $\tau^{-} \rightarrow \pi^{-} \nu$ the $\pi^{-}$is not isotropic in $\tau$ frame but rather travels in the backward direction. (Note that polarization of the secondary resonances, $\rho^{-}$and $a_{1}^{-}$, is not taken into account, i.e., tertiary pions are assumed to be isotropic in the rest frame of the parent resonance.) The lineshapes of intermediate resonances are generated in Gaussian forms.

For a given decay channel of the $\tau$, the output of the generator is a sequence of production-decay topologies (including tertiary pions from decays of secondary resonances) accompanied by weights that represent the computed matrix elements. Correspondingly, all distributions are plotted using these weights. At the moment, the individual hadrons of the primary hadron jet are not generated, and are treated as undetected. 
Thus generated $\tau$ events are then propagated through the detector using GEANT. Rather than fully digitize the response of electronic trackers (drift chambers and silicon microstrips), the individual hits are randomly distributed around central positions according to corresponding resolutions. Then, particle momentum is obtained by fitting the "smeared" trajectory in magnetic field. Likewise, the hits in individual cells of the EMCal are not fully simulated at this early stage. Instead, the energy and position of a shower are smeared according to aforementioned resolutions. The problem of overlapping showers is not yet addressed.

For a given decay channel of the $\tau$, all histograms are normalized to the total number of occurring decays (so that the net contents are just the accepted fraction of all $\tau$ decays in this channel). With this convention, the acceptance of a selection becomes immediately obvious without further normalization.

\subsection{Detecting the Decay $\tau^{-} \rightarrow \pi^{-} \nu$}

The momentum resolution for the $\pi^{-}$with $p_{\pi}>1 \mathrm{GeV}$ from the decay $\tau^{-} \rightarrow \pi^{-} \nu$, as illustrated in Fig. 3, on average amounts to some $2.7 \%$ for either detector. The unsmeared transverse mass, $M_{T}=\sqrt{m_{\pi}^{2}+p_{T}^{2}}+p_{T}$, is plotted for all generated $\tau^{-} \rightarrow \pi^{-} \nu$ events in Fig. 1. Also shown are the corresponding smeared distributions for those decay pions that pass the procedure-driven "default" selections in lab. momentum $\left(p_{\pi}>1 \mathrm{GeV}\right)$, in emission angle relative to incident beam $(\Theta<400 \mathrm{mrad})$, in kink angle $\left(\theta_{\tau \pi}>10 \mathrm{mrad}\right)$, and in transverse momentum to $\tau$ direction $\left(p_{T}>250 \mathrm{MeV}\right)$. The Jacobian cusp of the original $M_{T}$ distribution is but slightly degraded by apparatus smearings of the proposed COSMOS and TOSCA detectors. Therefore, in both detectors the produced $\tau$ leptons will be efficiently tagged by $\tau^{-} \rightarrow \pi^{-} \nu$ decays. 


\subsection{Detecting the Decay $\tau^{-} \rightarrow \rho^{-} \nu, \pi^{0} \rightarrow \gamma \gamma$}

In this subsection that deals with the decay $\tau^{-} \rightarrow \rho^{-} \nu$, the "default" selections for the $\pi^{-}$from $\rho^{-} \rightarrow \pi^{-} \pi^{0}$ (namely, $p_{\pi^{-}}>1 \mathrm{GeV}, \Theta>400 \mathrm{mrad}, \theta_{\tau \pi}>10 \mathrm{mrad}$, and $p_{T}^{\pi}>250$ $\mathrm{MeV}$ ) are implicitly included in all distributions and quoted acceptances. For the $\pi^{-}$, we use the values of emission angles as measured near the production point in emulsion.

We assume that the conversion point of the photon from $\pi^{0} \rightarrow \gamma \gamma$ is found and measured in emulsion and, therefore, its direction is precisely known. The energy of the parent photon is reconstructed by adding up the energies of unconverted daughter gammas (by hits in the EMCal) and of conversion electrons that are momentum-analyzed in the tracker.

In this scheme of reconstruction, there is a potential danger of double counting: a conversion electron, that has already been momentum-analyzed in the gap between the two targets, may shower in the next target producing further conversion electrons and gammas that should no longer be counted. We assume that, given a relatively large gap between successive targets, these "unwanted daughters" can be distinguished by their large displacement from the shower axis. Whether or not this can be done experimentally is currently under investigation, and our preliminary results are very encouraging. But for the moment, we realize this on GEANT level by "stopping" conversion electrons just before they hit the next target.

By either allowing or excluding double counting, we obtain two different estimates of the parent-photon energy. For either approach, the ratio $E_{\gamma}^{v i s} / E_{\gamma}^{\text {true }}$ between the estimated and true values of energy is plotted in Fig. 5 for photons originating from different

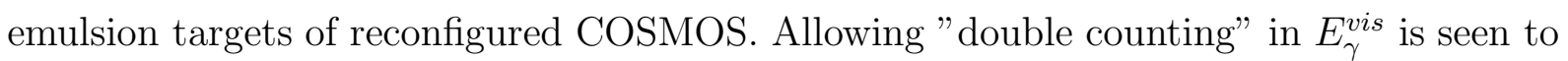


result in overestimating the true energy for a significant fraction of photons from $\pi^{0} \rightarrow \gamma \gamma$. Again for either estimate of $E_{\gamma}^{v i s}$, plotted in Fig. 6 is the visible two-photon mass $m_{\gamma \gamma}$ for events occurring in different targets of COSMOS. Quite predictably, "double counting" in $E_{\gamma}^{\text {vis }}$ degrades the $\pi^{0}$ signals in the two upstream targets. The very similar distributions of $E_{\gamma}^{\text {vis }} / E_{\gamma}^{\text {true }}$ and of $m_{\gamma \gamma}$ for different targets of reconfigured TOSCA are not illustrated.

With "double counting" excluded, the overall $m_{\gamma \gamma}$ distributions for the reconfigured COSMOS and TOSCA detectors are shown in Fig. 7. Fixing the mass window for $\pi^{0}$ selection requires detailed simulations of detector response to primary hadrons and to background processes, and therefore is impossible at this stage. For several realistic mass windows for $m_{\gamma \gamma}(135 \pm 25,20$, and $15 \mathrm{MeV})$, the acceptances of reconfigured detectors to $\tau^{-} \rightarrow \rho^{-} \nu$ decays are listed in Table 2 together with those of the original COSMOS detector [13]. (Note that reconstruction of $\pi^{0} \rightarrow \gamma \gamma$ decays, that is essential for selecting the $\tau^{-} \rightarrow \rho^{-} \nu$ decays, is virtually impossible in the baseline design of TOSCA [14.) Either of the proposed detectors is seen to have a substantially higher acceptance to $\tau^{-} \rightarrow \rho^{-} \nu$ than the baseline detector of COSMOS.

Tentatively selecting the $\pi^{0} \rightarrow \gamma \gamma$ decays in the $m_{\gamma \gamma}$ window of $135 \pm 25 \mathrm{MeV}$, we then plot the mass of the $\pi^{-} \pi^{0}$ system, as reconstructed in either detector (see Fig. 7). And finally, in Fig. Ð we plot the reconstructed transverse mass for $\tau^{-} \rightarrow \rho^{-} \nu$, $M_{T}=\sqrt{m_{\pi \pi}^{2}+p_{T}^{2}}+p_{T}$. As the Jacobian structure of the original $M_{T}$ distribution is not destroyed by apparatus smearings, we may conclude that both proposed detectors will efficiently tag $\tau$ leptons by reconstructing the $\tau^{-} \rightarrow \rho^{-} \nu$ decays. 


\subsection{Detecting the Decay $\tau^{-} \rightarrow a_{1}^{-} \nu, a_{1}^{-} \rightarrow \pi^{-} \pi^{+} \pi^{-}$}

This decay proceeds through an intermediate $\rho^{0}\left(a_{1}^{-} \rightarrow \rho^{0} \pi^{-}\right)$and has a branching fraction of $9.4 \%$. The three-prong decay channel is plagued by the background from three-prong collisions of secondary hadrons with the nuclei of emulsion, including coherent production of the $a_{1}$ state by pions. Still, it may be worthwhile to check whether or not the expected $M_{T}$ distribution is distinctive.

Using the procedure-driven selections of $p_{\pi}>1 \mathrm{GeV}$ and $\Theta<400 \mathrm{mrad}$ for all three pions and determining pion directions near the production point in emulsion, in Fig. 目 we plot the reconstructed transverse mass for the decay $\tau^{-} \rightarrow a_{1}^{-} \nu, a_{1}^{-} \rightarrow \pi^{-} \pi^{+} \pi^{-}$:

$M_{T}=\sqrt{m_{3 \pi}^{2}+p_{T}^{2}}+p_{T}$. (Note that no $\theta_{\tau \pi}$ selection is required for observing a threeprong decay in emulsion.) That the very distinct Jacobian structure of the original $M_{T}$ distribution persists in the smeared plots is very encouraging: backgrounds from pion dissociation and from coherent $a_{1}$ production should be amassed at much lower values of $M_{T}$. Whether or not the proposed detectors are good enough for separating the decays $\tau^{-} \rightarrow a_{1}^{-} \nu$ can only be decided by detailed comparisons with the simulated three-prong background.

\subsection{Detecting the decay $\tau^{-} \rightarrow e^{-} \nu \bar{\nu}$}

Of the decays that are not in the (quasi-)two-body category, we consider the decay $\tau^{-} \rightarrow e^{-} \nu \bar{\nu}$ that, compared to $\tau^{-} \rightarrow \mu^{-} \nu \bar{\nu}$, poses experimental problems due to electrons showering in thick emulsion. Owing to thinner emulsion targets and to good reconstruction of shower energy, we may detect the $\tau^{-} \rightarrow e^{-} \nu \bar{\nu}$ decays more efficiently than the baseline apparata of COSMOS and TOSCA. 
In reconstructing the electron from $\tau^{-} \rightarrow e^{-} \nu \bar{\nu}$, we follow a strategy analogous to the one used for photons: the energy is reassembled from pieces, using magnetic analysis for all positive and negative electrons leaving the emulsion and hits in the EMCal for the unconverted daughter gammas. Thus reconstructed energy is denoted as $E_{e}^{v i s}$. Then, we are able to estimate the transverse momentum with respect to $\tau$ direction as $p_{T}^{v i s}=$ $E_{e}^{v i s} \sin \theta_{\tau e}$, where $\theta_{\tau e}$ is the angle of the observed kink.

That the sign of the generic electron be correctly assigned is important for rejecting the background from semileptonic decays of charm produced by neutrinos. Therefore, the $e^{-}$track must be followed in emulsion down to the exit and reliably linked to electronic trackers. For this to be possible, we assume that the energy of the generic- $e^{-}$track at the exit point from the target of origin, $E_{t r a c k}^{\text {exit }}$, must exceed $1 \mathrm{GeV}$. In a thick target, the generic $e^{-}$may fail this requirement by losing too much energy through bremsstrahlung, even though the total energy of the shower can be estimated.

Thus, a candidate event for $\tau^{-} \rightarrow e^{-} \nu \bar{\nu}$ must satisfy the following conditions: $E_{\text {track }}^{\text {exit }}>$ $1 \mathrm{GeV}, \Theta_{e}<400 \mathrm{mrad}, p_{T}^{v i s}>250 \mathrm{MeV}$, and $\theta_{\tau e}>10 \mathrm{mrad}$. For the $\tau^{-} \rightarrow e^{-} \nu \bar{\nu}$ events that survive these minimum selections in the detector, we plot the ratio between the reconstructed and original energies of the $e^{-}, E_{e}^{v i s} / E_{e}^{\text {true }}$, in Fig. 8. The reconstruction of electron energy is good in both detectors proposed. For either the reconfigured and baseline designs of COSMOS and TOSCA, the fractions of all generated $\tau^{-} \rightarrow e^{-} \nu \bar{\nu}$ events that survive the above selections are listed in Table 3. The reconfigured detectors are seen to have higher acceptances to the decay $\tau^{-} \rightarrow e^{-} \nu \bar{\nu}$ than the baseline schemes for COSMOS and TOSCA. 


\section{Summary}

The large- $\Delta m^{2}$ domain of $\nu_{\mu} \rightarrow \nu_{\tau}$ oscillations may be further probed by the next generation of short-baseline neutrino experiments using the emulsion technique for detecting $\tau$ decays: COSMOS at Fermilab and TOSCA at CERN. In this paper, we propose alternative schemes of these experiments that emphasize good spectrometry for charged particles and for electromagnetic showers and efficient reconstruction of $\pi^{0} \rightarrow \gamma \gamma$ decays. Either configuration features a sequence of relatively thin emulsion targets, immersed in magnetic field and interspersed with electronic trackers, and a fine-grained electromagnetic calorimeter built of lead glass. These elements act as an integral whole in reconstructing the electromagnetic showers. This conceptual scheme shows superior performance in identifying the $\tau$ (quasi-)two-body decays by their characteristic kinematics and in selecting the electronic decays of the $\tau$. 


\section{References}

[1] B. Pontecorvo, Zh. Eksp. Teor. Fiz. 7, 172 (1958); Sov. Phys. JETP 6, 429 (1958).

[2] M. C. Gonzales-Garcia, H. Nunokawa, O. L. G. Peres, T. Stanev, and J. W. F. Valle, Update on Atmospheric Neutrinos, Phys. Rev. D58, 033004 (1998).

[3] C. Athanassopoulos et al., LSND Coll., Phys. Rev. Lett. 77, 3082 (1996).

[4] E. Ables et al., MINOS Coll., Fermilab Proposal P875 (1995).

[5] P.F. Harrison, D.H. Perkins, and W.G. Scott, Phys. Lett. B349, 137 (1995).

[6] A. Acker and S. Pakvasa, preprint hep-ph//9611423 (1996).

[7] M. Gell-Mann, P. Ramond, and R. Slansky, in Supergravity, eds. P. van Nieuwenhuizen and D. Freedman (North Holland, Amsterdam, 1979), p. 315.

[8] N. Ushida et al., E531 Coll., Phys. Rev. Lett. 57, 2897 (1986).

[9] M. de Jong et al., CHORUS Coll., preprint CERN-PPE/93-131 (1993); E. Eskut et al., CHORUS Coll., Nucl. Instr. and Meth. A401, 7 (1997).

[10] E. Eskut et al., et al., CHORUS Coll., Phys. Lett. B424, 202 (1998); E. Eskut et al., CHORUS Coll., preprint CERN-PPE/98-73 (1998).

[11] J. Altegoer et al., NOMAD Coll., Nucl. Instr. and Meth. A404, 96 (1988); J. Altegoer et al., NOMAD Coll., preprint CERN-EP/98-57 (1998).

[12] K. Kodama et al., E803/COSMOS Coll., Fermilab Proposal P803 (1993).

[13] K. Kodama et al., E803/COSMOS Coll., Update Report on Fermilab E803/COSMOS (1995). 
[14] A.S. Ayan et al., TOSCA Coll., Letter of Intent, CERN-SPSC/97-5, SPSC/I213 (March 1997).

[15] K. Kodama et al., E653 Coll., preprint DPNU-96-33 (June 1996).

[16] R. Rameika, A. Asratyan, M. Balatz, G. Davidenko, A. Dolgolenko, G. Dzyubenko, V. Kaftanov, M. Kubantsev, A. Sitnikov, V. Verebryusov, and V. Vishnyakov, LeadGlass Option for the COSMOS Electromagnetic Calorimeter, E803/COSMOS internal report (February 1996).

[17] S. Aoki et al., Nucl. Instr. and Meth. B51, 466 (1990). 


\begin{tabular}{|c|c|c|}
\hline Quantity Compared & COSMOS & TOSCA \\
\hline Proton energy, GeV & 120 & 350 \\
\hline Protons per cycle & $4 \cdot 10^{13}$ & $6 \cdot 10^{13}$ \\
\hline Cycle time, s & 1.9 & 19.2 \\
\hline Protons per year & $3.7 \cdot 10^{20}$ & $4.3 \cdot 10^{19}$ \\
\hline Length of decay channel, $\mathrm{m}$ & 800 & 414 \\
\hline Distance from target to detector, $\mathrm{m}$ & 960 & 806 \\
\hline Emulsion mass, $\mathrm{kg}$ & 865 & 2784 \\
\hline Emulsion area, $\mathrm{m}^{2}$ & $1.80 \times 1.40$ & $1.44 \times 1.44$ \\
\hline Emulsion thickness, $\mathrm{cm}$ & $2 \times 4.5$ & $6 \times 6.0$ \\
\hline$\langle E\rangle$ of $\nu_{\mu}$ beam (by flux), $\mathrm{GeV}$ & 12 & 25 \\
\hline$\langle E\rangle$ of $\nu_{\mu}$-induced CC events, $\mathrm{GeV}$ & 18 & 37 \\
\hline$\langle E\rangle$ of $\nu_{\tau}$-induced CC events, $\mathrm{GeV}$ & 24 & 54 \\
\hline$\langle L / E\rangle, \mathrm{km} / \mathrm{GeV}$ & 0.023 & 0.012 \\
\hline$\left\langle\sigma\left(\nu_{\tau}\right)\right\rangle /\left\langle\sigma\left(\nu_{\mu}\right)\right\rangle$ & 0.27 & 0.50 \\
\hline$\Sigma(\mathrm{BR} \times$ efficiency $)$ & 0.114 & 0.104 \\
\hline Total number of $\nu_{\mu}$-induced CC events & $8.1 \cdot 10^{6}$ & $6.0 \cdot 10^{6}$ \\
\hline Total number of background events & 1.3 & 1.3 \\
\hline Total background events, 90\% CL & 3.5 & 3.5 \\
\hline Reach in $\sin ^{2}(2 \theta)$ for large $\Delta m^{2}$ & $2.8 \cdot 10^{-5}$ & $1.5 \cdot 10^{-5}$ \\
\hline Reach in $\Delta m^{2}$ for maximum mixing, $\mathrm{eV}^{2}$ & 0.10 & 0.10 \\
\hline
\end{tabular}

Table 1: General parameters of the baseline designs of COSMOS [13] and TOSCA [14]. 


\begin{tabular}{|c|c|c|c|}
\hline $\begin{array}{c}\text { Mass window for } \\
\text { selecting the } \pi^{0}\end{array}$ & $\begin{array}{c}\text { Our design } \\
\text { for COSMOS }\end{array}$ & $\begin{array}{c}\text { Our design } \\
\text { for TOSCA }\end{array}$ & $\begin{array}{c}\text { Baseline design } \\
\text { for COSMOS }\end{array}$ \\
\hline Unrestricted & 0.40 & 0.42 & 0.31 \\
$135 \pm 25 \mathrm{MeV}$ & 0.31 & 0.28 & 0.19 \\
$135 \pm 20 \mathrm{MeV}$ & 0.29 & 0.25 & 0.18 \\
$135 \pm 15 \mathrm{MeV}$ & 0.26 & 0.21 & 0.16 \\
\hline
\end{tabular}

Table 2: The fractions of all $\tau^{-} \rightarrow \rho^{-} \nu, \rho^{-} \rightarrow \pi^{-} \pi^{0}$ decays that survive different $\pi^{0}$ selections (as indicated) and the "default" selections for the $\pi^{-}$. 


\begin{tabular}{|c|c|c|c|}
\hline $\begin{array}{c}\text { Our design } \\
\text { for COSMOS }\end{array}$ & $\begin{array}{c}\text { Our design } \\
\text { for TOSCA }\end{array}$ & $\begin{array}{c}\text { Baseline design } \\
\text { for COSMOS }\end{array}$ & $\begin{array}{c}\text { Baseline design } \\
\text { for TOSCA }\end{array}$ \\
\hline 0.55 & 0.56 & 0.31 & 0.42 \\
\hline
\end{tabular}

Table 3: The fractions of all $\tau^{-} \rightarrow e^{-} \nu \bar{\nu}$ decays that survive the minimum selections in the detector, as detailed in the text. 


\section{Figure Captions}

Fig. 1. The configurations proposed for COSMOS (top) and TOSCA (bottom). Overlaid are the $\tau^{-} \rightarrow \rho^{-} \nu$ decays in either detector.

Fig. 2. The computed energy spectra of $\nu_{\tau}$-induced CC events in COSMOS (a) and TOSCA (b). The absolute normalization is arbitrary.

Fig. 3. In the decay $\tau^{-} \rightarrow \pi^{-} \nu$, the ratio between the measured and true values of $\pi^{-}$ momentum, $R=p_{\pi}^{v i s} / p_{\pi}^{\text {true }}$, for the proposed schemes of COSMOS (a) and TOSCA (b). Here and in all subsequent figures, the smearing due to Coulomb scattering in emulsion is fully taken into account.

Fig. $₫$. Transverse mass $M_{T}=\sqrt{p_{T}^{2}+m_{h}^{2}}+p_{T}$ for the (quasi-)two-body decays $\tau^{-} \rightarrow h^{-} \nu$ with $h^{-}=\pi^{-}$(left-hand column), $h^{-}=\rho^{-} \rightarrow \pi^{-} \pi^{0}$ (middle column), and $h^{-}=a_{1}^{-} \rightarrow \pi^{-} \pi^{+} \pi^{-}$(right-hand column). The unsmeared $M_{T}$ distributions for all generated events in each channel are shown in the top row. The smeared distributions for events detected by the reconfigured COSMOS and TOSCA are shown in the middle and bottom rows, respectively.

Fig. 5. The ratio $R=E_{\gamma}^{\text {vis }} / E_{\gamma}^{\text {true }}$ between the estimated and true values of energy for photons originating from different emulsion targets of reconfigured COSMOS, with "double counting" in $E_{\gamma}^{v i s}$ either allowed (the top row) or forbidden (the bottom row). The three columns stand for the three targets of the proposed detector.

Fig. 6. The reconstructed two-photon mass $m_{\gamma \gamma}$ for the $\pi^{0} \rightarrow \gamma \gamma$ decays occurring in different emulsion targets of reconfigured COSMOS, with "double counting" in $E_{\gamma}^{\text {vis }}$ either allowed (the top row) or forbidden (the bottom row). The three columns stand for the three targets of the proposed detector. 
Fig. 7. The masses $m_{\gamma \gamma}$ (top row) and $m_{\pi \pi}$ (bottom row), as reconstructed in the reconfigured COSMOS and TOSCA detectors (the left-hand and right-hand columns, respectively) excluding double counting in $E_{\gamma}^{v i s}$. Note that the lineshape of the original $\rho^{-}$has been generated as a Gaussian with $\sigma=75 \mathrm{MeV}$.

Fig. 8. The ratio between the reconstructed and original energies of the $e^{-}$from $\tau^{-} \rightarrow e^{-} \nu \bar{\nu}, R=E_{e}^{v i s} / E_{e}^{t r u e}$, for the proposed configurations of COSMOS (a) and TOSCA (b). 

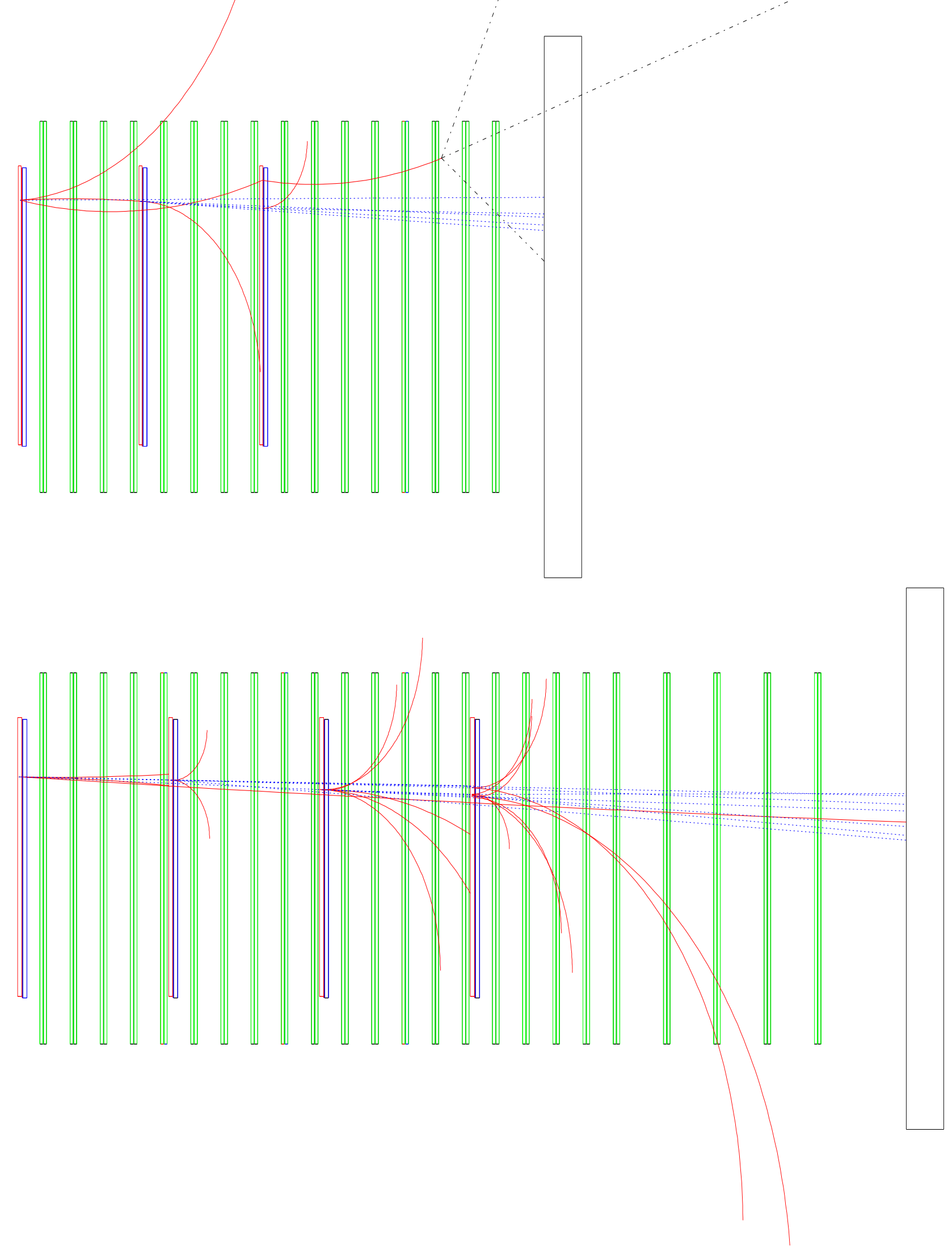

Figure 1: The configurations proposed for COSMOS (top) and TOSCA (bottom). Overlaid are the $\tau^{-} \rightarrow \rho^{-} \nu$ decays in either detector. 

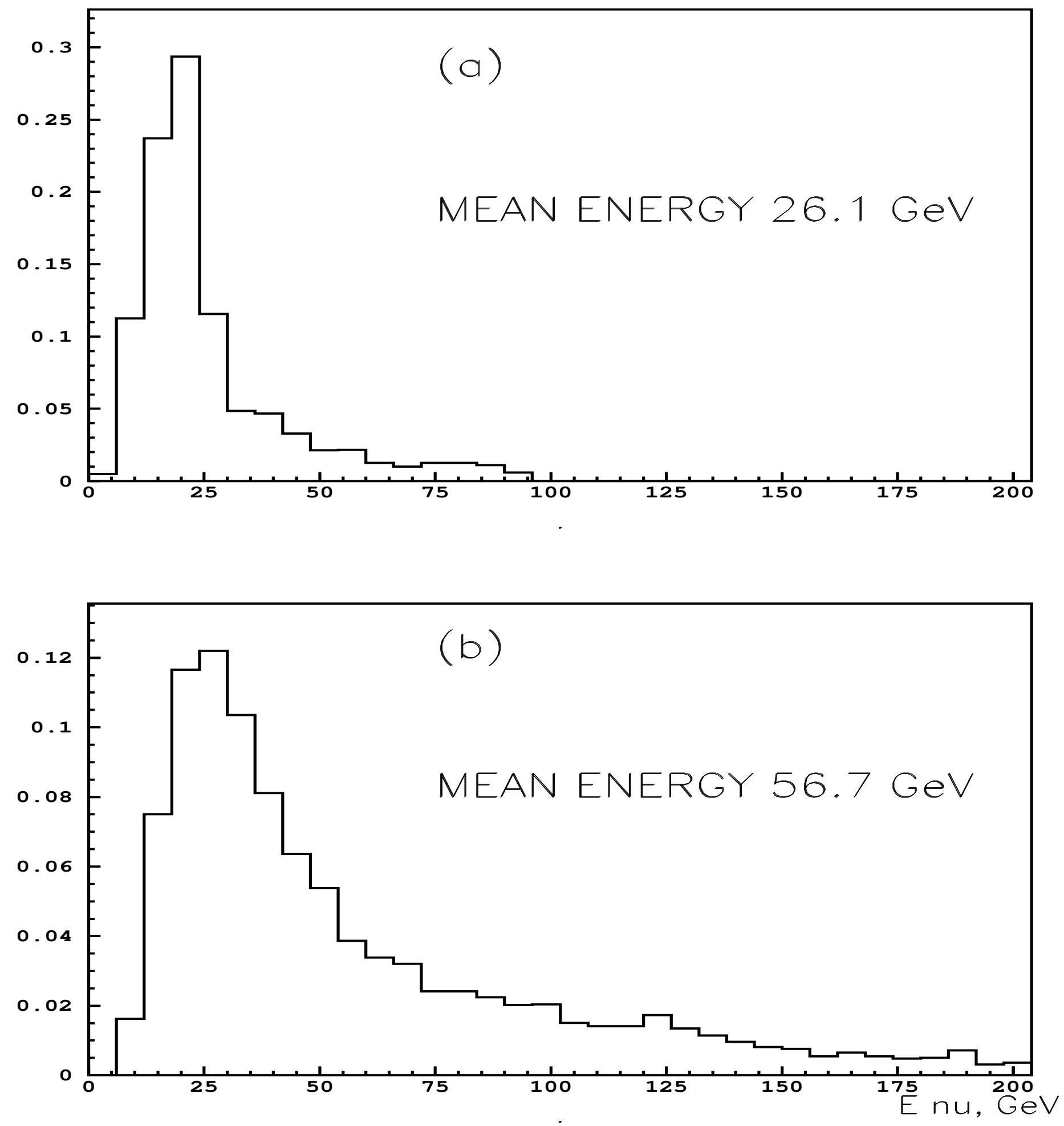

Figure 2: The computed energy spectra of $\nu_{\tau}$-induced CC events in COSMOS (a) and TOSCA (b). The absolute normalization is arbitrary. 

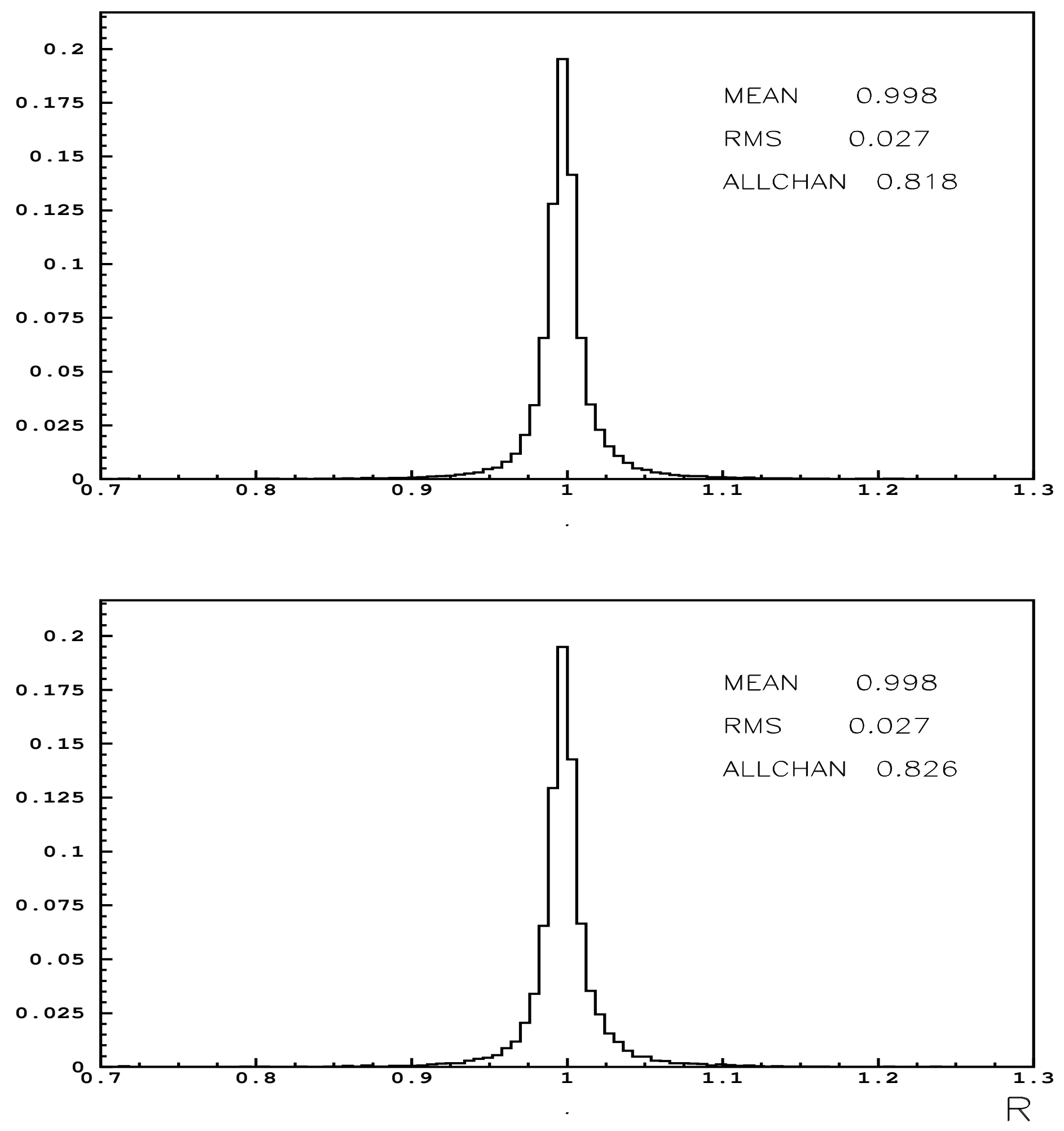

Figure 3: In the decay $\tau^{-} \rightarrow \pi^{-} \nu$, the ratio between the measured and true values of $\pi^{-}$ momentum, $R=p_{\pi}^{v i s} / p_{\pi}^{\text {true }}$, for the proposed schemes of COSMOS (a) and TOSCA (b). Here and in all subsequent figures, the smearing due to Coulomb scattering in emulsion is fully taken into account. 

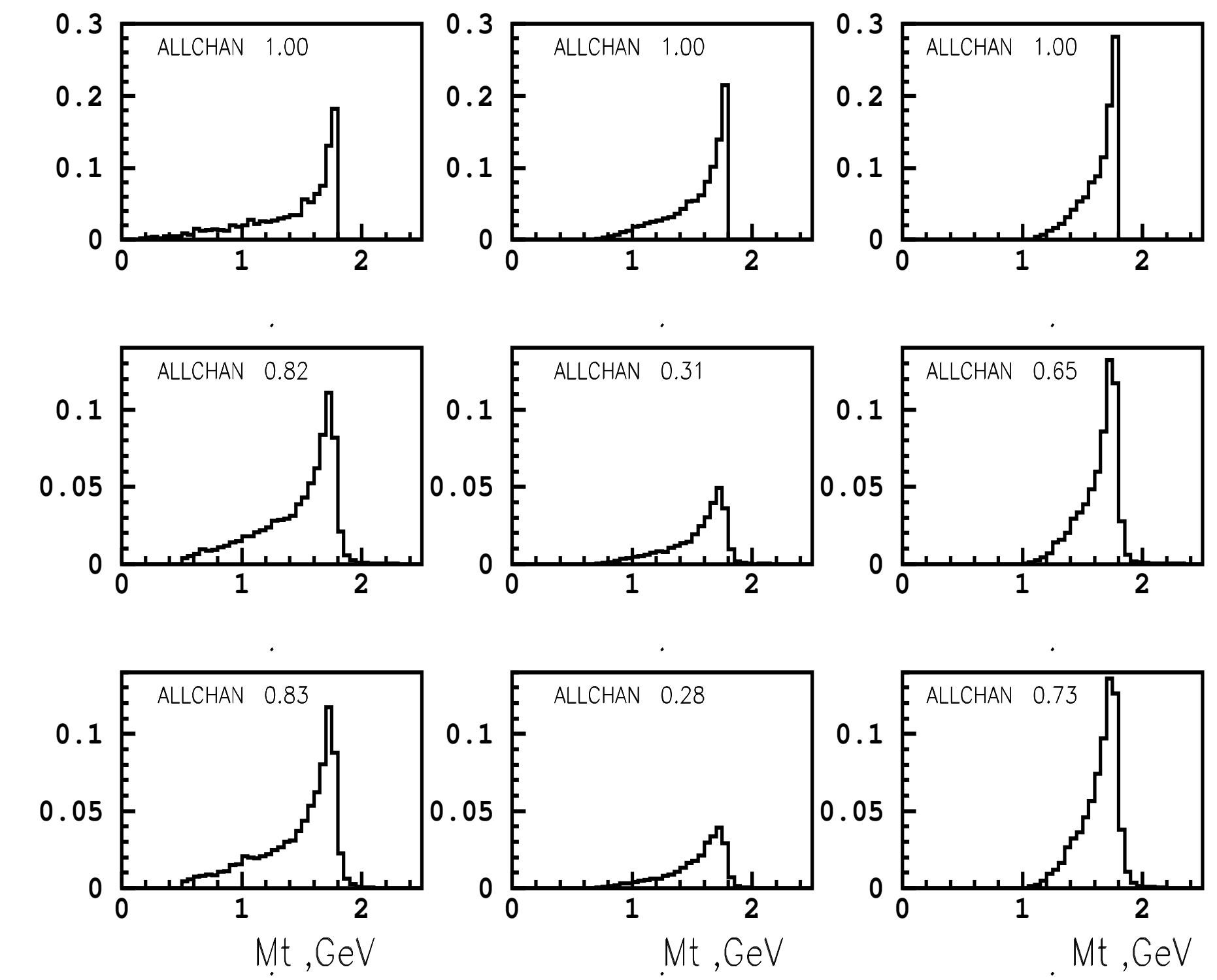

Figure 4: Transverse mass $M_{T}=\sqrt{p_{T}^{2}+m_{h}^{2}}+p_{T}$ for the (quasi-)two-body decays $\tau^{-} \rightarrow$ $h^{-} \nu$ with $h^{-}=\pi^{-}$(left-hand column), $h^{-}=\rho^{-} \rightarrow \pi^{-} \pi^{0}$ (middle column), and $h^{-}=$ $a_{1}^{-} \rightarrow \pi^{-} \pi^{+} \pi^{-}$(right-hand column). The unsmeared $M_{T}$ distributions for all generated events in each channel are shown in the top row. The smeared distributions for events detected by the reconfigured COSMOS and TOSCA are shown in the middle and bottom rows, respectively. 
TARGET 1
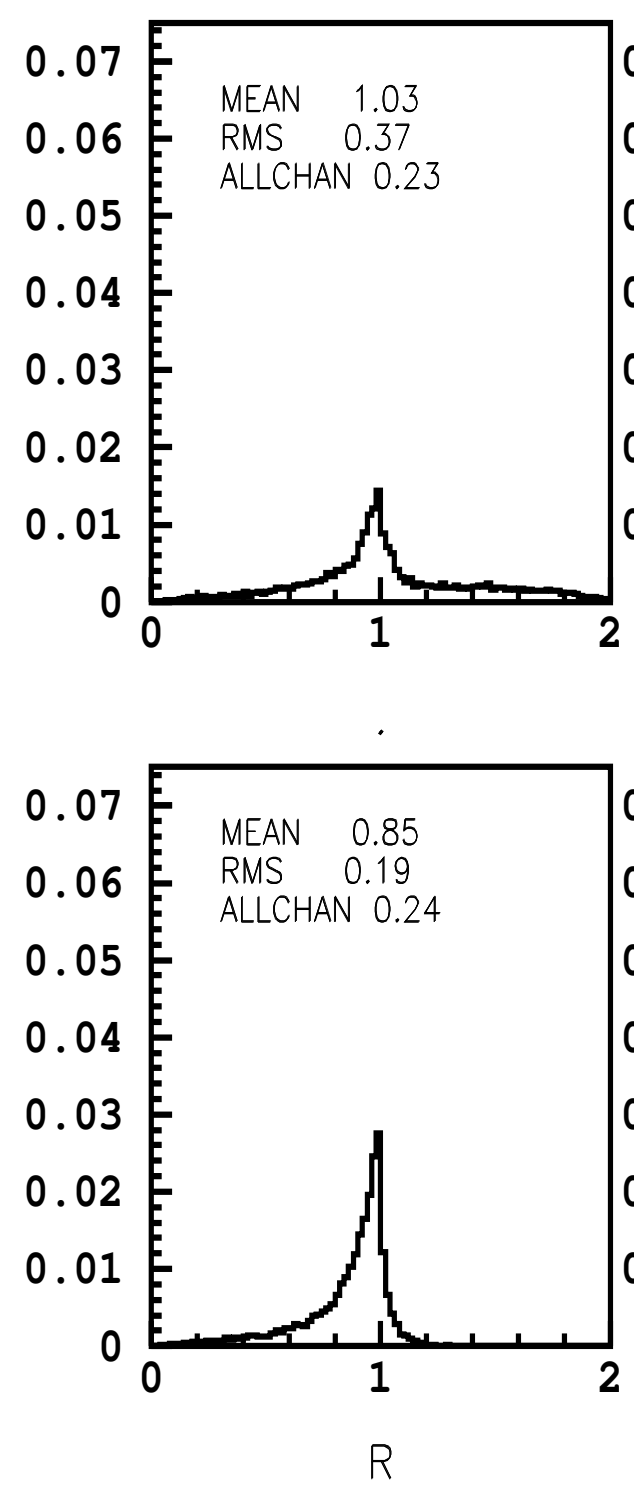

TARGET 2
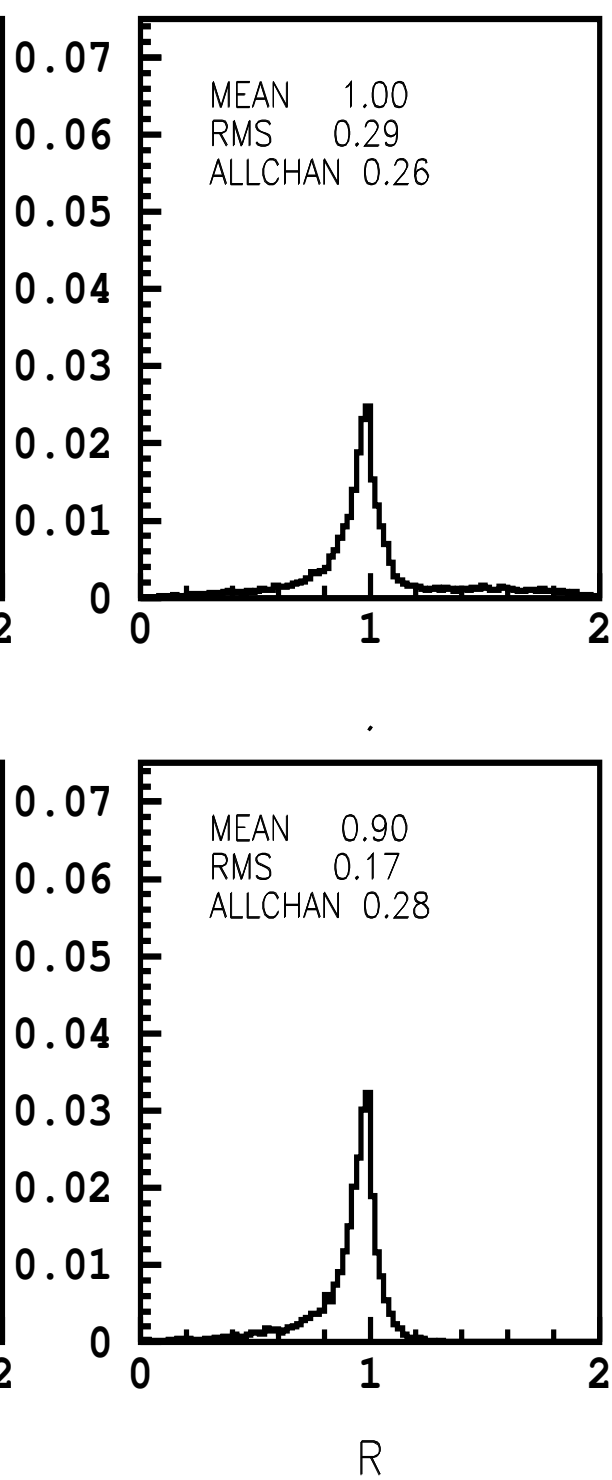

TARGET 3
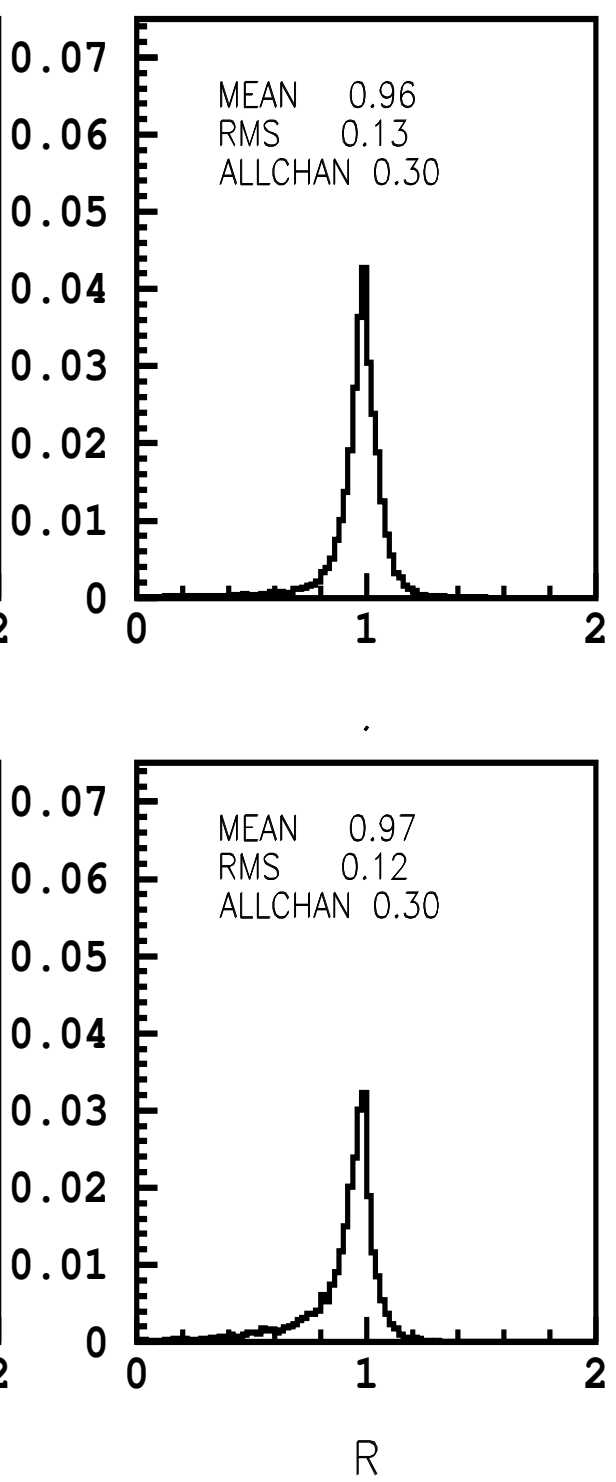

Figure 5: The ratio $R=E_{\gamma}^{v i s} / E_{\gamma}^{\text {true }}$ between the estimated and true values of energy for photons originating from different emulsion targets of reconfigured COSMOS, with "double counting" in $E_{\gamma}^{v i s}$ either allowed (the top row) or forbidden (the bottom row). The three columns stand for the three targets of the proposed detector. 
TARGET 1

TARGET 2

TARGET 3
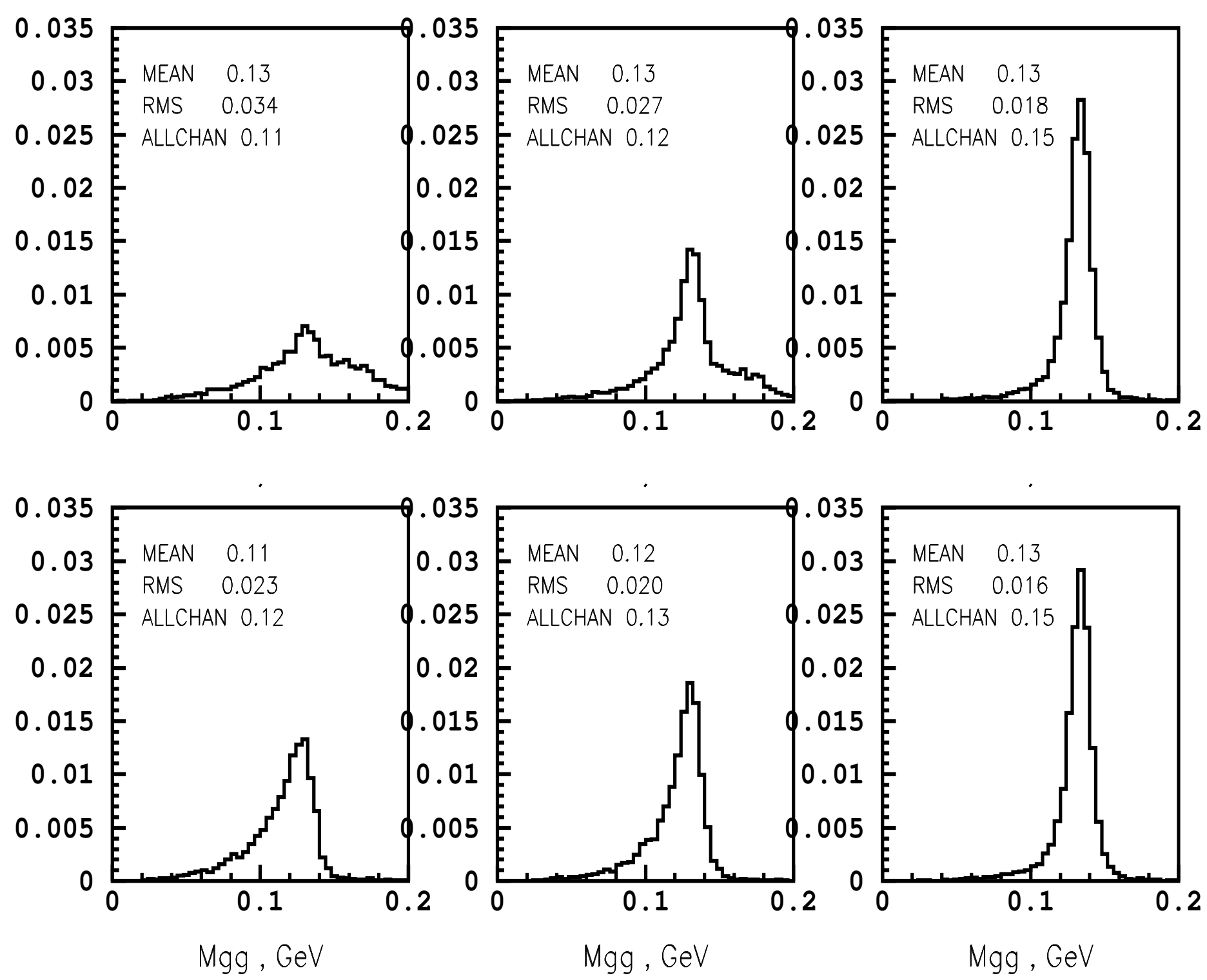

Figure 6: The reconstructed two-photon mass $m_{\gamma \gamma}$ for the $\pi^{0} \rightarrow \gamma \gamma$ decays occurring in different emulsion targets of reconfigured COSMOS, with "double counting" in $E_{\gamma}^{v i s}$ either allowed (the top row) or forbidden (the bottom row). The three columns stand for the three targets of the proposed detector. 

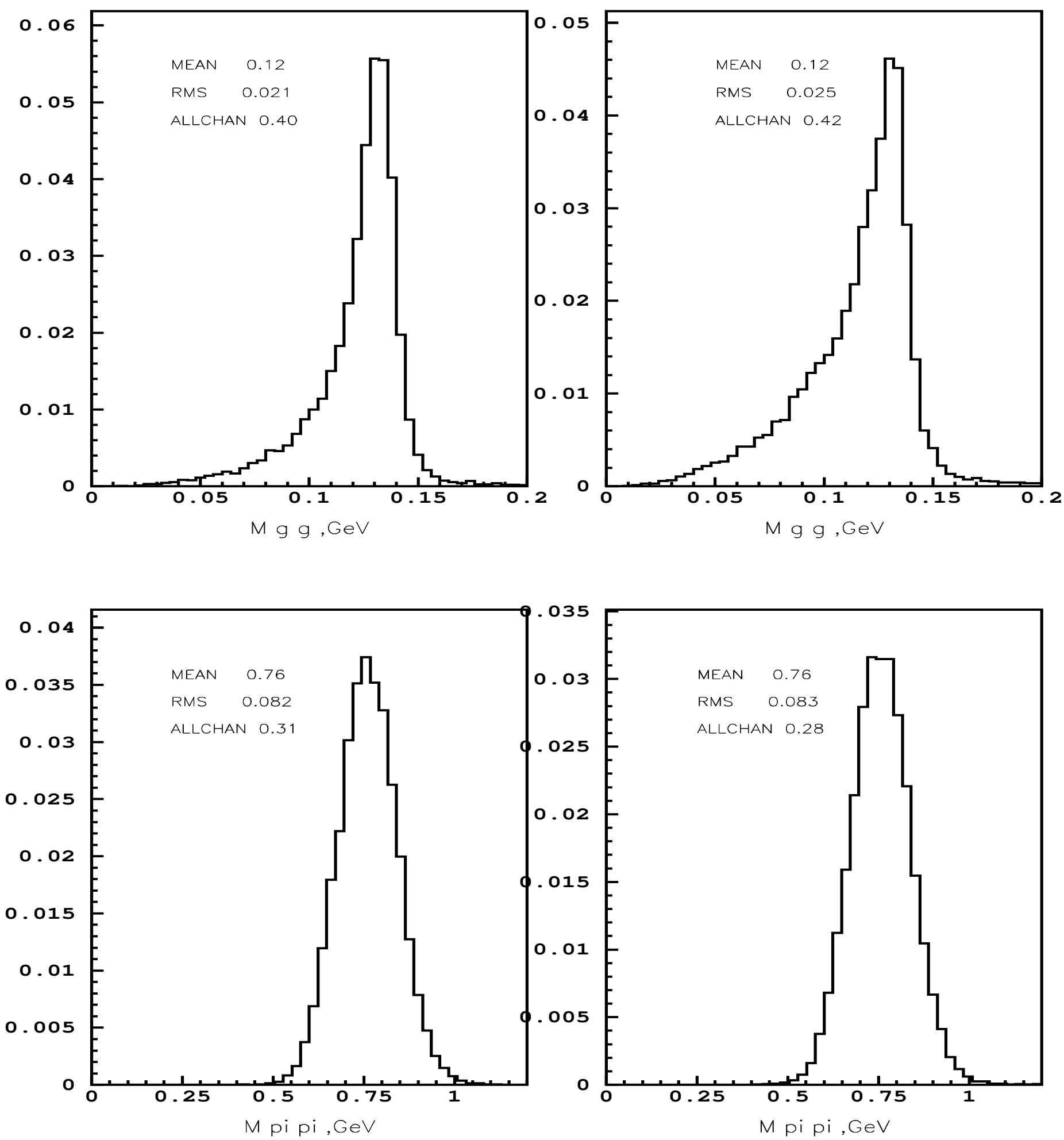

Figure 7: The masses $m_{\gamma \gamma}$ (top row) and $m_{\pi \pi}$ (bottom row), as reconstructed in the reconfigured COSMOS and TOSCA detectors (the left-hand and right-hand columns, respectively) excluding double counting in $E_{\gamma}^{v i s}$. Note that the lineshape of the original $\rho^{-}$has been generated as a Gaussian with $\sigma=75 \mathrm{MeV}$. 

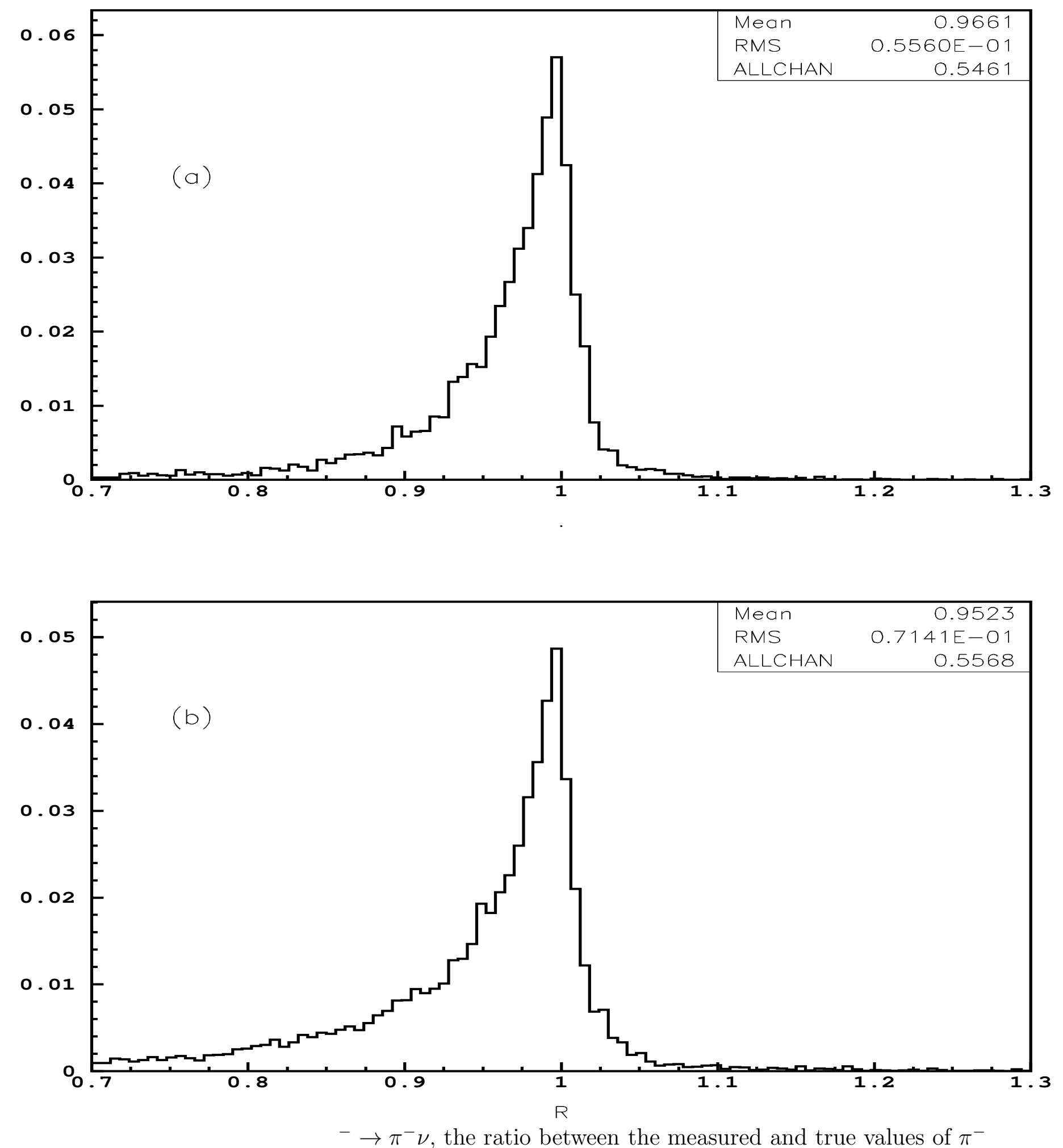

FingmurenBunthe the deris $x p_{\pi}^{\text {true }}$, for the proposed schemes of COSMOS (a) and TOSCA (b).

Here and in all subsequent figures, the smearing due to Coulomb scattering in emulsion is fully taken into account. 

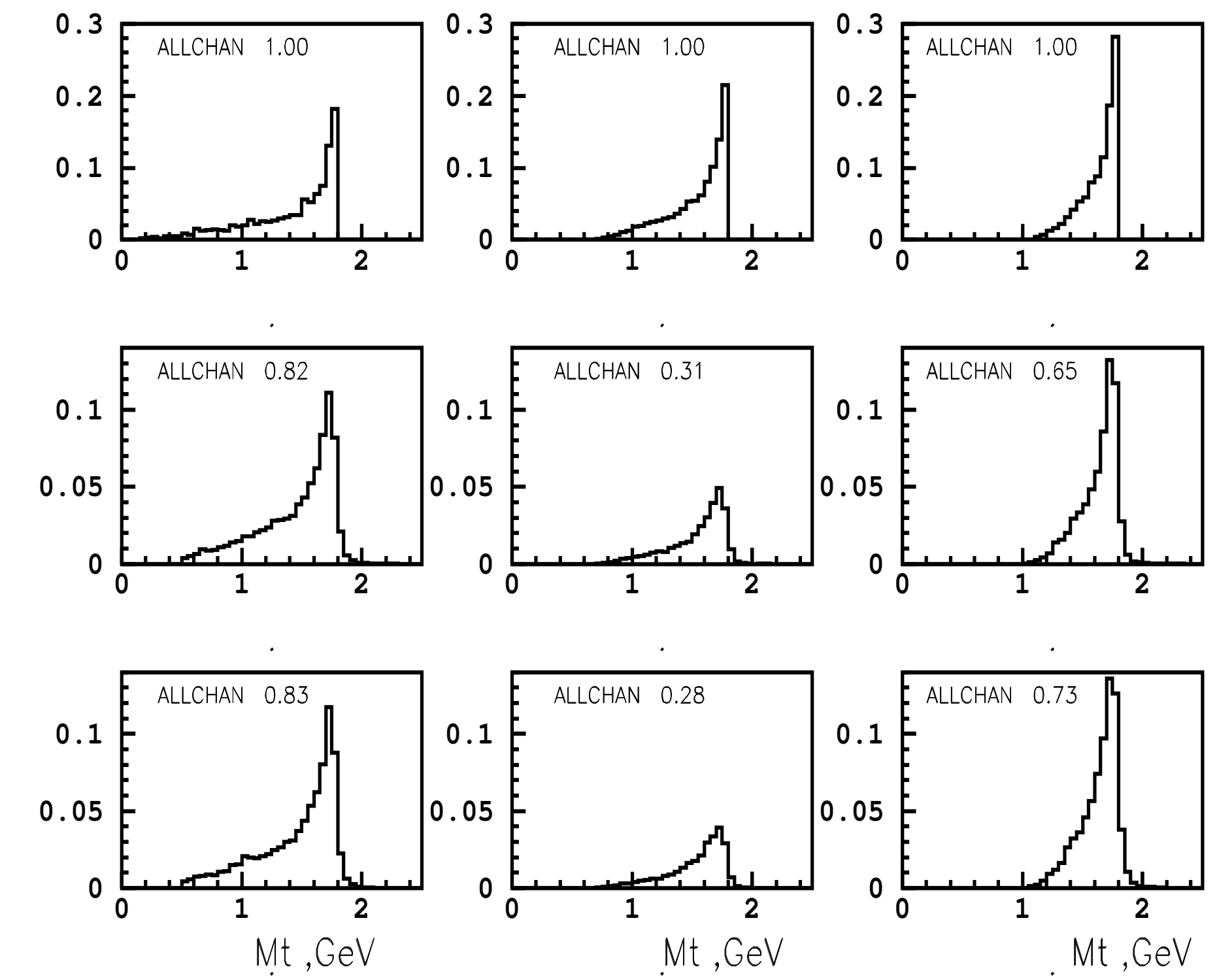

Figure 4: Transverse mass $M_{T}=\sqrt{p_{T}^{2}+m_{h}^{2}}+p_{T}$ for the (quasi-)two-body decays $\tau^{-} \rightarrow$ $h^{-} \nu$ with $h^{-}=\pi^{-}$(left-hand column), $h^{-}=\rho^{-} \rightarrow \pi^{-} \pi^{0}$ (middle column), and $h^{-}=$ $a_{1}^{-} \rightarrow \pi^{-} \pi^{+} \pi^{-}$(right-hand column). The unsmeared $M_{T}$ distributions for all generated events in each channel are shown in the top row. The smeared distributions for events detected by the reconfigured COSMOS and TOSCA are shown in the middle and bottom rows, respectively. 
TARGET 1
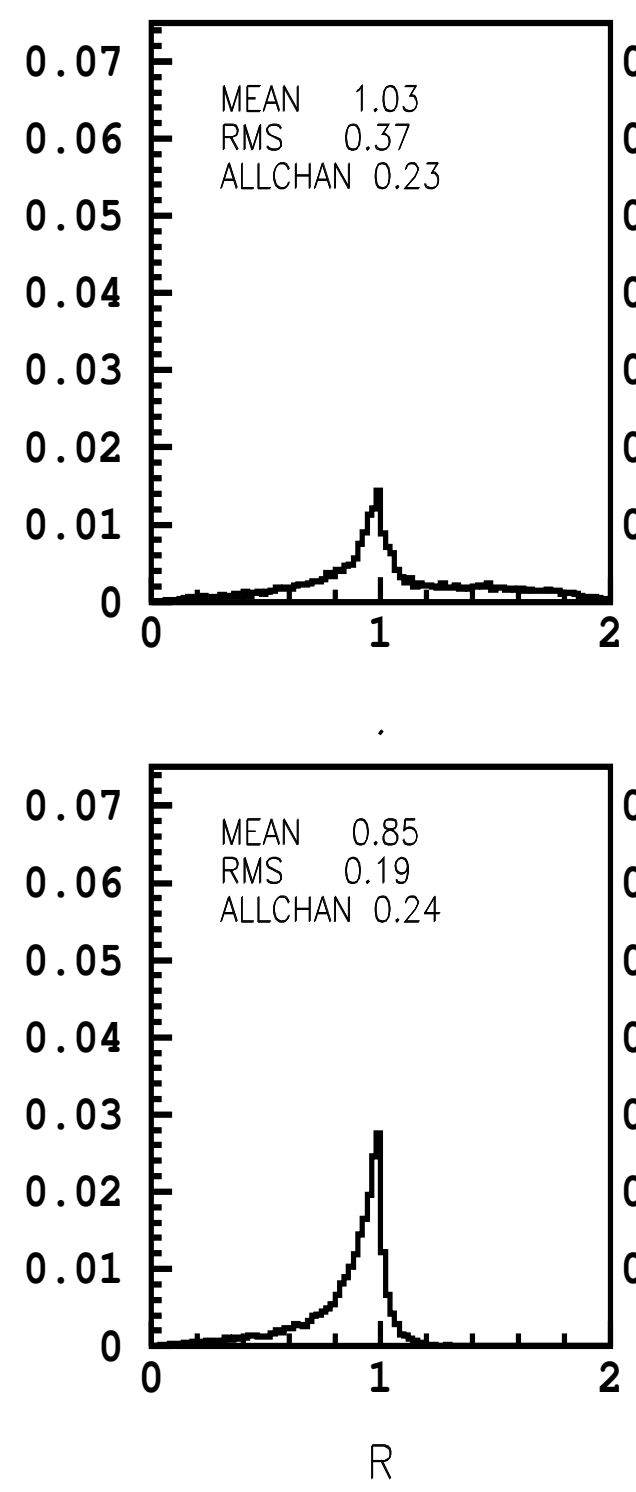

TARGET 2
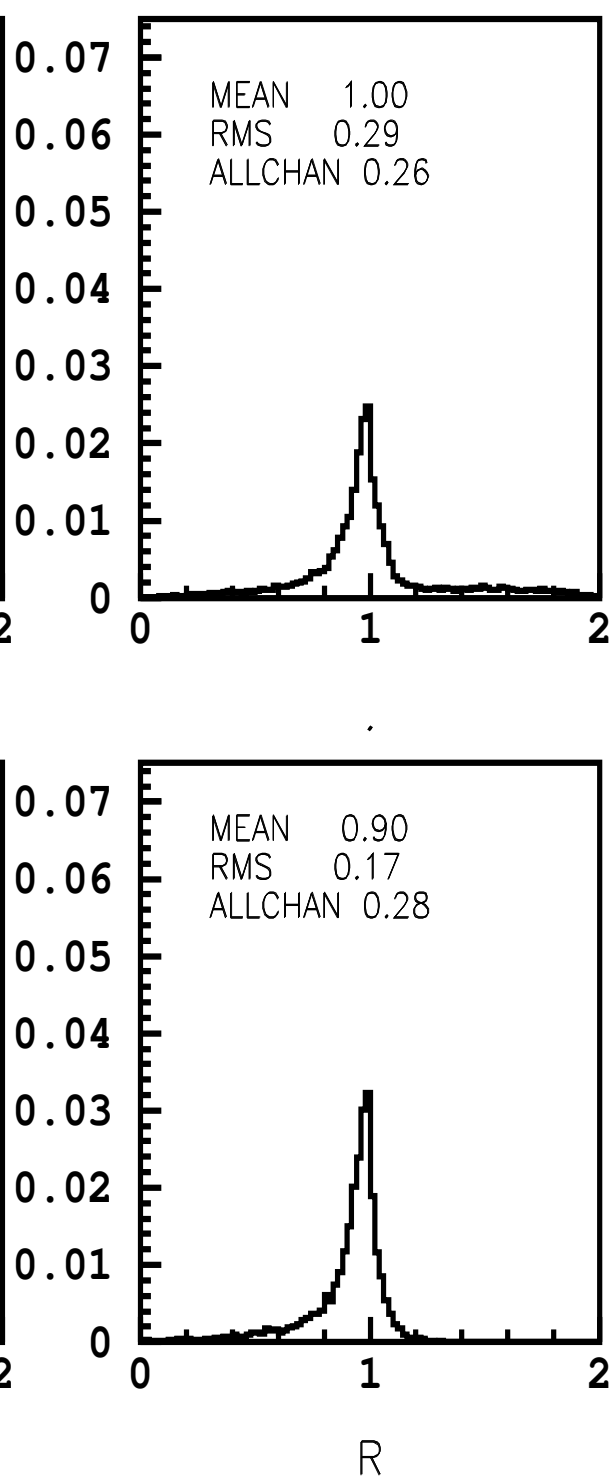

TARGET 3
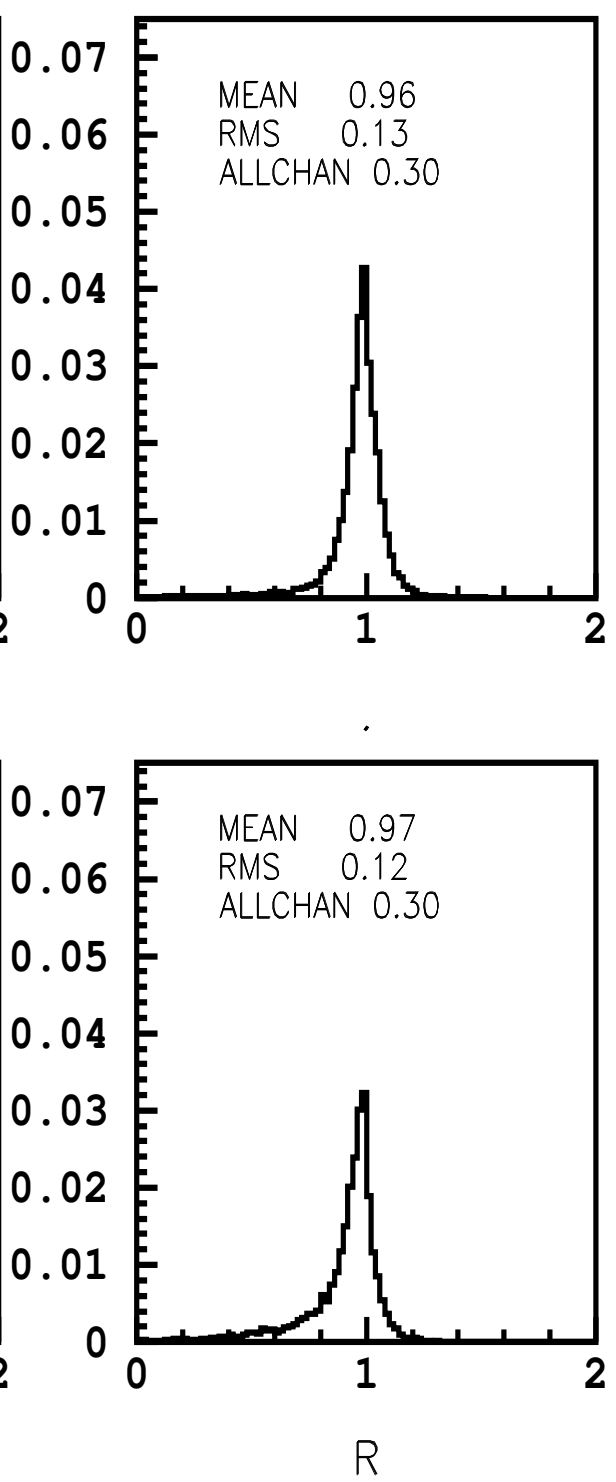

Figure 5: The ratio $R=E_{\gamma}^{v i s} / E_{\gamma}^{\text {true }}$ between the estimated and true values of energy for photons originating from different emulsion targets of reconfigured COSMOS, with "double counting" in $E_{\gamma}^{v i s}$ either allowed (the top row) or forbidden (the bottom row). The three columns stand for the three targets of the proposed detector. 
TARGET 1

TARGET 2

TARGET 3
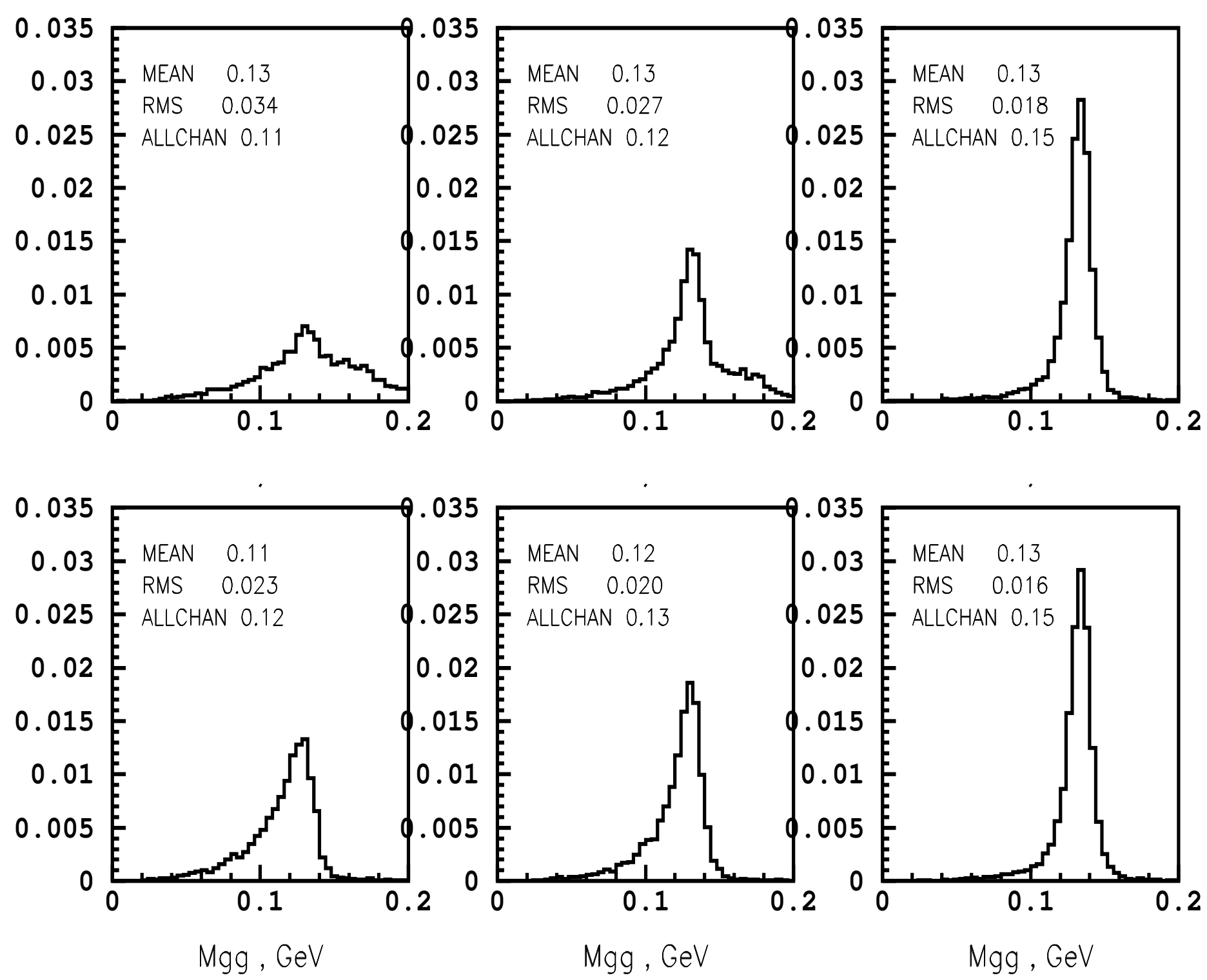

Figure 6: The reconstructed two-photon mass $m_{\gamma \gamma}$ for the $\pi^{0} \rightarrow \gamma \gamma$ decays occurring in different emulsion targets of reconfigured COSMOS, with "double counting" in $E_{\gamma}^{v i s}$ either allowed (the top row) or forbidden (the bottom row). The three columns stand for the three targets of the proposed detector. 

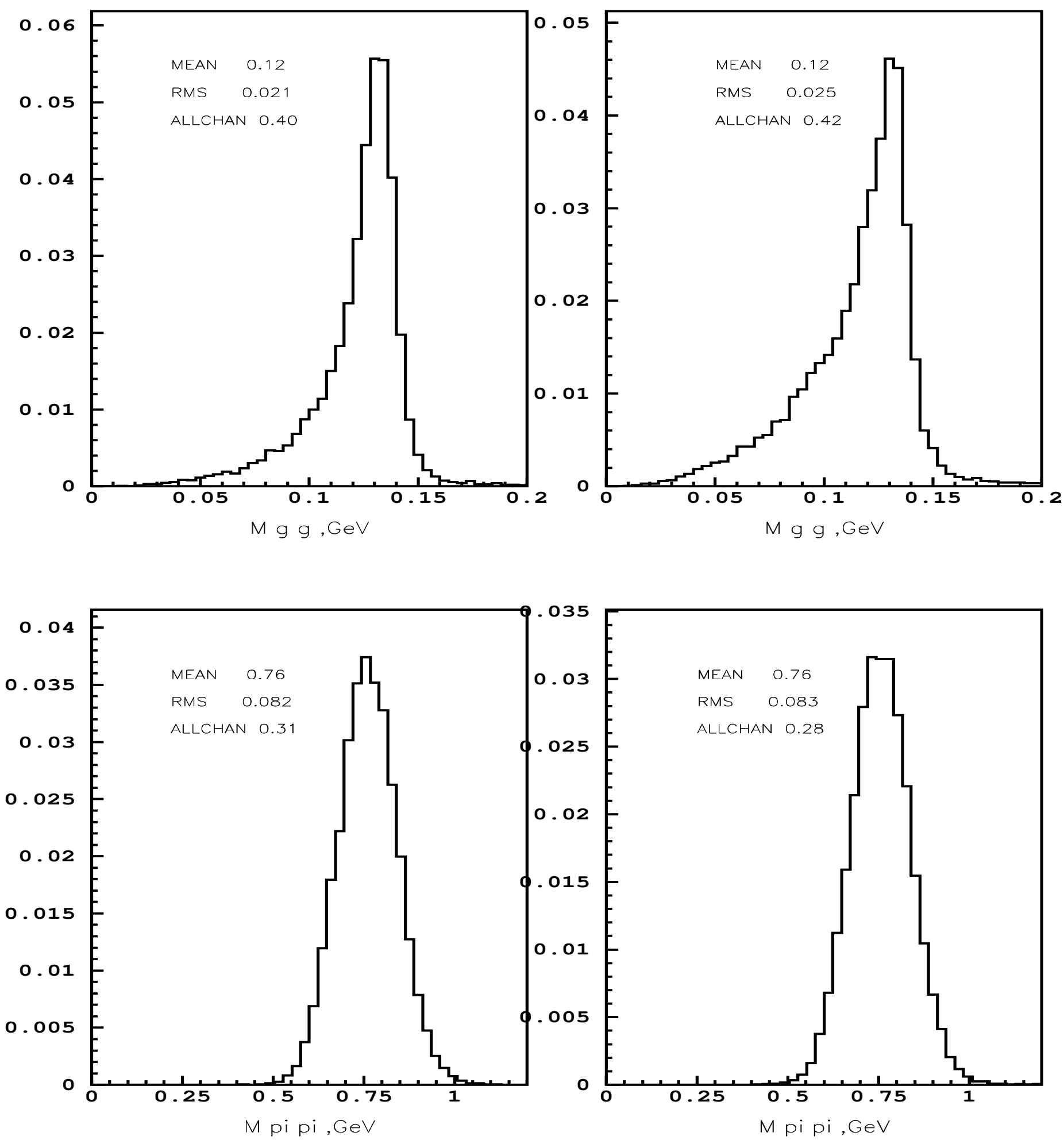

Figure 7: The masses $m_{\gamma \gamma}$ (top row) and $m_{\pi \pi}$ (bottom row), as reconstructed in the reconfigured COSMOS and TOSCA detectors (the left-hand and right-hand columns, respectively) excluding double counting in $E_{\gamma}^{v i s}$. Note that the lineshape of the original $\rho^{-}$has been generated as a Gaussian with $\sigma=75 \mathrm{MeV}$. 

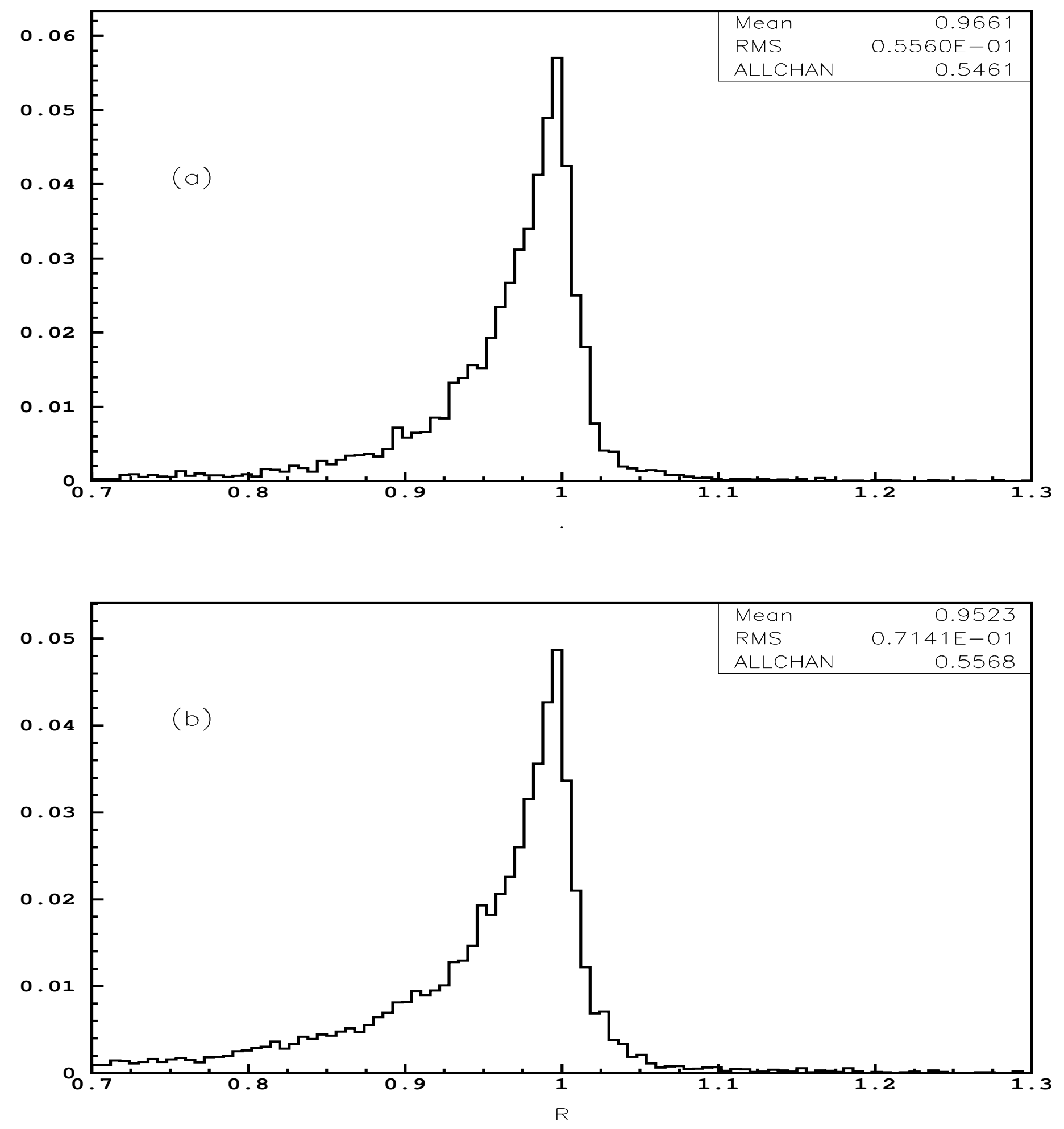

Figure 8: The ratio between the reconstructed and original energies of the $e^{-}$from $\tau^{-} \rightarrow$ $e^{-} \nu \bar{\nu}, R=E_{e}^{v i s} / E_{e}^{\text {true }}$, for the proposed configurations of COSMOS (a) and TOSCA (b). 